\title{
A Semigroup Expansion for Pricing Barrier Options
}

\author{
Takashi Kato, ${ }^{1}$ Akihiko Takahashi, ${ }^{2}$ and Toshihiro Yamada ${ }^{2,3}$ \\ ${ }^{1}$ Division of Mathematical Science for Social Systems, Graduate School of Engineering Science, \\ Osaka University, 1-3, Machikaneyama-cho, Toyonaka, Osaka 560-8531, Japan \\ ${ }^{2}$ Graduate School of Economics, The University of Tokyo, 7-3-1 Hongo, Bunkyo, Tokyo 113-0033, Japan \\ ${ }^{3}$ Mitsubishi UFJ Trust Investment Technology Institute Co., Ltd. (MTEC), 2-6, Akasaka 4-Chome, Minato, Tokyo 107-0052, Japan \\ Correspondence should be addressed to Toshihiro Yamada; toshihiro.ymd@gmail.com
}

Received 20 April 2014; Accepted 11 August 2014; Published 14 September 2014

Academic Editor: Lukasz Stettner

Copyright (C) 2014 Takashi Kato et al. This is an open access article distributed under the Creative Commons Attribution License, which permits unrestricted use, distribution, and reproduction in any medium, provided the original work is properly cited.

This paper presents a new asymptotic expansion method for pricing continuously monitoring barrier options. In particular, we develop a semigroup expansion scheme for the Cauchy-Dirichlet problem in the second-order parabolic partial differential equations (PDEs) arising in barrier option pricing. As an application, we propose a concrete approximation formula under a stochastic volatility model and demonstrate its validity by some numerical experiments.

\section{Introduction}

Since the Merton's seminal work [1] barrier options have been quite popular and important products in both academics and financial business for the last four decades. In particular, fast and accurate computation of their prices and Greeks is highly desirable in the risk management, which is a tough task under the finance models commonly used in practice. Thus, it has been one of the central issues in the mathematical finance community. Among various approaches to attacking the problem, this paper proposes a new semigroup expansion scheme under general diffusion setting.

Firstly, let us note that the value of a continuously monitoring down-and-out barrier option is expressed as the following form under the so-called risk-neutral probability measure:

$$
\begin{aligned}
C_{\text {Barrier }}(T, x) & =\mathrm{E}\left[e^{-\int_{0}^{T} c\left(X_{r}^{x}\right) d r} f\left(X_{T}^{x}\right) 1_{\{\tau>T\}}\right] \\
& =\mathrm{E}\left[e^{-\int_{0}^{T} c\left(X_{r}^{x}\right) d r} f\left(X_{T}^{x}\right) 1_{\left\{\min _{t \in[0, T\}} X_{t}>L\right\}}\right] .
\end{aligned}
$$

Here, $T>0$ is a maturity of the option and $f$ is an option payoff function at maturity. $\left(X_{t}^{x}\right)_{t}$ denotes a vector process with initial value $x$ under the risk-neutral probability measure, which includes a price process of the underlying asset (usually given as the solution of a certain stochastic differential equation (SDE)). Also, $c$ stands for the risk-free interest rate process. Moreover, $L$ stands for a constant lower barrier, that is $L<x$, and $\tau$ is the hitting time to $L$ :

$$
\tau=\inf \left\{t \in[0, T]: X_{t}^{x} \leq L\right\} .
$$

It is well-known that a possible approach in computation of $C_{\text {Barrier }}(T, x)$ is the Euler-Maruyama scheme, which stores the sample paths of the process $\left(X_{t}^{x}\right)_{t}$ through an $n$-time discretization with the step size $T / n$. When applying this scheme to pricing a continuously monitoring barrier option, one kills the simulated process, say $\left(\bar{X}_{t_{i}}^{x}\right)_{i}$ if $\bar{X}_{t_{i}}^{x}$ exits from the domain $(L, \infty)$ until the maturity $T$. The usual EulerMaruyama scheme is suboptimal since it does not control the diffusion paths between two successive dates $t_{i}$ and $t_{i+1}$ : the diffusion paths could have crossed the barriers and come back to the domain without being detected. It is also known that the error between $C_{\mathrm{Barrier}}(T, x)$ and $\bar{C}_{\mathrm{Barrier}}(T, x)$, the barrier option price obtained by the Euler-Maruyama scheme is of order $\sqrt{T / n}$, as opposed to the order $T / n$ for standard plainvanilla options (see [2]). Thus, various Monte-Carlo schemes have been proposed for improving the order of the error (see [3] for instance).

One of the other tractable approaches for calculating $C_{\text {Barrier }}(T, x)$ is to derive an analytical approximation. If we obtain an accurate approximation formula, it is a powerful 
tool for pricing continuously monitoring barrier options because we need not rely on Monte-Carlo simulations anymore. However, from a mathematical viewpoint, deriving an approximation formula by applying stochastic analysis is not an easy task since the Malliavin calculus cannot be directly applied. It is due to the nonexistence of the Malliavin derivative $D_{t} \tau$ (see [4]) and to the fact that the minimum (maximum) process of the Brownian motion has only the first-order differentiability in the Malliavin sense. Thus, neither approach in [5] nor in [6] can be applied directly to evaluation of continuously monitoring barrier options, while they are applicable to pricing discrete barrier options (see [7] for the detail).

This paper proposes a new general method for the approximation of barrier option prices. Particularly, our objective is to price barrier options when the vector process of the underlying state variables is described by the following perturbed SDE under a filtered complete probability space with the risk-neutral probability measure, which will be concretely defined in the begging of the next section:

$$
\begin{gathered}
d X_{t}^{\varepsilon, x}=b\left(X_{t}^{\varepsilon, x}, \varepsilon\right) d t+\sigma\left(X_{t}^{\varepsilon, x}, \varepsilon\right) d B_{t}, \\
X_{0}^{\varepsilon, x}=x,
\end{gathered}
$$

where $\varepsilon$ is a small parameter. In this case, the barrier option price (1) is characterized as a solution of the Cauchy-Dirichlet problem:

$$
\begin{aligned}
\frac{\partial}{\partial t} u^{\varepsilon}(t, x)+\mathscr{L}^{\varepsilon} u^{\varepsilon}(t, x) & =0, \quad(t, x) \in[0, T) \times(L, \infty), \\
u^{\varepsilon}(T, x) & =f(x), \quad x>L, \\
u^{\varepsilon}(t, L) & =0, \quad t \in[0, T],
\end{aligned}
$$

where the differential operator $\mathscr{L}^{\varepsilon}$ is determined by the diffusion coefficients $b$ and $\sigma$ with the risk-free interest rate $c$, which will be explicitly defined in the next section.

Next, we introduce an asymptotic expansion formula:

$$
\begin{aligned}
u^{\varepsilon}(t, x)= & u^{0}(t, x)+\varepsilon v_{1}^{0}(t, x)+\cdots+\varepsilon^{n-1} v_{n-1}^{0}(t, x) \\
& +O\left(\varepsilon^{n}\right)
\end{aligned}
$$

where $O$ denotes the Landau symbol. The function $u^{0}(t, x)$ is the solution of (4) with $\varepsilon=0$ : if $b(x, 0)$ and $\sigma(x, 0)$ have some simple forms such as constants (as in the BlackScholes model), we already know the closed form of $u^{0}(t, x)$ and hence obtain the price. Then, we are able to get the approximate value for $u^{\varepsilon}(t, x)$ through evaluation of the coefficient functions $v_{1}^{0}(t, x), \ldots, v_{n-1}^{0}(t, x)$. In fact, they are also characterized as the solution of a certain PDE with the Dirichlet condition. By formal asymptotic expansions, (5) above and the following,

$$
\mathscr{L}^{\varepsilon}=\mathscr{L}^{0}+\varepsilon \widetilde{\mathscr{L}}_{1}^{0}+\cdots+\varepsilon^{n-1} \widetilde{\mathscr{L}}_{n-1}^{0}+\cdots,
$$

we can derive the following PDE which $v_{k}^{0}(t, x)$ satisfies:

$$
\begin{gathered}
\frac{\partial}{\partial t} v_{k}^{0}(t, x)+\mathscr{L}^{0} v_{k}^{0}(t, x)+g_{k}^{0}(t, x)=0, \\
(t, x) \in[0, T) \times(L, \infty), \\
v_{k}^{0}(T, x)=0, \quad x>L, \\
v_{k}^{0}(t, L)=0, \quad t \in[0, T],
\end{gathered}
$$

where $g_{k}^{0}(t, x)$ will be given explicitly in Section 2. Moreover, by applying the Feynman-Kac approach to the PDE (7), we obtain a semigroup representation of $v_{k}^{0}$. That is, for each $k=$ $1, \ldots, n-1$,

$$
\begin{aligned}
& v_{k}^{0}(T-t, x) \\
& =\sum_{l=1}^{k} \sum_{\left(\beta^{i}\right)_{i=1}^{l}} \sum_{\mathbb{N}^{l}, \sum_{i} \beta^{i}=k} \int_{0}^{t} \int_{0}^{t_{1}} \cdots \int_{0}^{t_{l-1}} P_{t-t_{1}}^{D} \widetilde{\mathscr{L}}_{\beta^{1}}^{0} P_{t_{1}-t_{2}}^{D} \\
& \times \widetilde{\mathscr{L}}_{\beta^{2}}^{0} \cdots P_{t_{l-1}-t_{l}}^{D} \widetilde{\mathscr{L}}_{\beta^{l}}^{0} \\
& \times P_{t_{l}}^{D} f(x) d t_{l} \cdots d t_{1},
\end{aligned}
$$

where $\left(P_{t}^{D}\right)_{t}$ is a semigroup defined in Section 2. We will justify the above argument in a mathematically rigorous manner in Section 2.

The theory of the Cauchy-Dirichlet problem for this kind of the second order parabolic PDE is well understood for the case of bounded domains (see [8-10] e.g.). As for an unbounded domain case such as (4), [11] provides the existence and uniqueness results for a solution of the PDE and the Feynman-Kac type formula, the part of which will be cited as Theorem 1 in Section 2. However, some mathematical difficulty exists for applying the results of [11] to the PDE (7). More precisely, the function $g_{k}^{0}(t, x)$ may be divergent at $t=T$. Hence, in order to obtain an asymptotic expansion (5), we generalize the result of [11] and the argument of the Feynman-Kac representation. Furthermore, we derive a new representation (8) for $v_{k}^{0}(t, x)$ by using the semigroup $\left(P_{t}^{D}\right)_{t}$. We notice that such a form is convenient for evaluation of $v_{k}^{0}(t, x)$ in concrete examples.

We also apply our method to pricing a barrier option in a stochastic volatility model. Then, as an example of (8) we obtain a new approximation formula of the barrier option price $C_{\mathrm{Barrier}}^{\mathrm{SV}, \varepsilon}$ under a stochastic volatility model as follows: for the initial value of the logarithmic underlying price $x$, the maturity $T$ and the lower barrier $L$,

$$
\begin{aligned}
C_{\text {Barrier }}^{\mathrm{SV}, \varepsilon}\left(T, e^{x}\right) & =\mathrm{E}\left[e^{-c T} f\left(S_{T}^{\varepsilon, x}\right) 1_{\left\{\min _{0 \leq t \leq T} S_{t}^{\varepsilon}>L\right\}}\right] \\
& \simeq P_{T}^{D} \bar{f}(x)+\varepsilon \int_{0}^{T} P_{T-r}^{D} \widetilde{\mathscr{L}}_{1}^{0} P_{r}^{D} \bar{f}(x) d r,
\end{aligned}
$$

where $\left(S_{t}^{\varepsilon, x}\right)_{t}$ is the underlying asset price process, $f$ is a payoff function and $\bar{f}(x)=f\left(e^{x}\right)$ and the expectation is taken under the risk-neutral probability measure. Here, $P_{T}^{D} \bar{f}(x)$ is regarded as the down-and-out barrier option price 
in the Black-Scholes model. Moreover, we confirm practical validity of our method through a numerical example given in Section 3.

Finally, we remark that there exist the previous works on barrier option pricing such as [12-15], which start with some specific models (e.g., Black-Scholes model or some type of fast mean-reversion model), and derive approximation formulas for discretely or continuously monitoring barrier option prices. Our approach is to firstly develop a general semigroup expansion scheme for the Cauchy-Dirichlet problem under multidimensional diffusion setting; then as an application, we provide a new approximation formula under a certain class of stochastic volatility model.

The organization of this paper is as follows: the next section firstly prepares the existence and uniqueness result for the Cauchy-Dirichlet problem in the second-order parabolic PDE associated with barrier option pricing. Then, we present our main result for an asymptotic expansion of barrier option prices. Section 3 shows numerical examples under a stochastic volatility model. Section 4 concludes. Finally, Appendix provides the proofs of the results in the main text.

\section{Asymptotic Expansion of Barrier Option Price}

2.1. Main Results. This subsection states the setup and our main results. The relevant assumptions $(\mathrm{A})-(\mathrm{H})$ will be explained in the next subsection.

Suppose first that a filtered complete probability space $\left(\Omega, \mathscr{F}, Q,\left\{\mathscr{F}_{t}\right\}_{t \in[0, T]}\right)$ is given, where $Q$ denotes the riskneutral probability measure, the filtration $\left\{\mathscr{F}_{t}\right\}_{t \in[0, T]}$ satisfies the usual conditions, and $T>0$ is some fixed time horizon. Then, the $d$-dimensional underlying state variable is described by the following perturbed SDE:

$$
\begin{gathered}
d X_{t}^{\varepsilon, x}=b\left(X_{t}^{\varepsilon, x}, \varepsilon\right) d t+\sigma\left(X_{t}^{\varepsilon, x}, \varepsilon\right) d B_{t}, \\
X_{0}^{\varepsilon, x}=x
\end{gathered}
$$

where $B$ is an $m$-dimensional Brownian motion and $\varepsilon$ is a small parameter. Let $b: \mathbb{R}^{d} \times I \rightarrow \mathbb{R}^{d}$ and $\sigma: \mathbb{R}^{d} \times I \rightarrow$ $\mathbb{R}^{d} \otimes \mathbb{R}^{m}$ be Borel measurable functions $(d, m \in \mathbb{N})$ where $I$ is an interval on $\mathbb{R}$ including the origin 0 , for instance $I=(-1,1)$.

We consider the SDE (10) for any $x \in \mathbb{R}^{d}$ and $\varepsilon \in I$. We note that Assumption (A) introduced in the next subsection guarantees the existence and uniqueness of a solution of (10). We also remark that at least one element of $X^{\varepsilon, x}$ stands for the underlying asset price process of a barrier option.

Next, we are interested in evaluation of the following barrier option price: for a small $\varepsilon$,

$$
\begin{aligned}
& \mathcal{u}^{\varepsilon}(t, x) \\
& =\mathrm{E}\left[\exp \left(-\int_{0}^{T-t} c\left(X_{r}^{\varepsilon, x}, \varepsilon\right) d r\right)\right. \\
& \left.\quad \times f\left(X_{T-t}^{\varepsilon, x}\right) 1_{\left\{\tau_{D}\left(X^{\varepsilon, x}\right) \geq T-t\right\}}\right], \quad(t, x) \in[0, T] \times \bar{D}
\end{aligned}
$$

for Borel measurable functions $f: \mathbb{R}^{d} \rightarrow \mathbb{R}, c: \mathbb{R}^{d} \times I \rightarrow \mathbb{R}$ and a domain $D \subset \mathbb{R}^{d}$.

Also, $\bar{D} \subset \mathbb{R}^{d}$ is the closure of $D$ and $\tau_{D}(w), w \in$ $C\left([0, T] ; \mathbb{R}^{d}\right)$ stands for the first exit time from $D$; that is

$$
\tau_{D}(w)=\inf \{t \in[0, T] ; w(t) \notin D\} .
$$

We also remark that the expectation operator $E[\cdot]$ is taken under the risk-neutral probability measure $Q$ and that $e^{-\int_{0}^{T-t} c\left(X_{r}^{\varepsilon, x}, \varepsilon\right) d r}$ represents the discount factor with the riskfree interest rate process $c$.

Let us define a second order differential operator $\mathscr{L}^{\varepsilon}$ by

$$
\mathscr{L}^{\varepsilon}=\frac{1}{2} \sum_{i, j=1}^{d} a^{i j}(x, \varepsilon) \frac{\partial^{2}}{\partial x^{i} \partial x^{j}}+\sum_{i=1}^{d} b^{i}(x, \varepsilon) \frac{\partial}{\partial x^{i}}-c(x, \varepsilon),
$$

where $a^{i j}=\sum_{k=1}^{d} \sigma^{i k} \sigma^{j k}$. We consider the following CauchyDirichlet problem for a PDE of parabolic type:

$$
\begin{gathered}
\frac{\partial}{\partial t} u^{\varepsilon}(t, x)+\mathscr{L}^{\varepsilon} u^{\varepsilon}(t, x)=0, \quad(t, x) \in[0, T) \times D, \\
u^{\varepsilon}(T, x)=f(x), \quad x \in D, \\
u^{\varepsilon}(t, x)=0, \quad(t, x) \in[0, T] \times \partial D .
\end{gathered}
$$

Under the assumptions $(\mathrm{A})-(\mathrm{E})$ stated in the next subsection, we have the following existence and uniqueness result due to Theorem 3.1 in [11].

Theorem 1. Assume $(A)-(E)$ which are given in Section 2.2. For each $\varepsilon \in I, u^{\varepsilon}(t, x)$ defined with formula (11) is a (classical) solution of (14) and

$$
\frac{\sup _{(t, x) \in[0, T] \times \bar{D}}\left|\mathcal{u}^{\varepsilon}(t, x)\right|}{1+|x|^{2 m}}<\infty .
$$

Moreover, if $w^{\varepsilon}(t, x)$ is also a solution of (14) satisfying the growth condition

$$
\frac{\sup _{(t, x) \in[0, T] \times \bar{D}}\left|w^{\varepsilon}(t, x)\right|}{1+|x|^{2 m^{\prime}}}<\infty
$$

for some $m^{\prime} \in \mathbb{N}$, then $u^{\varepsilon}=w^{\varepsilon}$.

Our main purpose is to present an asymptotic expansion of the barrier option price $u^{\varepsilon}(t, x)$ :

$$
\begin{aligned}
u^{\varepsilon}(t, x)= & u^{0}(t, x)+\varepsilon v_{1}^{0}(t, x)+\cdots \\
& +\varepsilon^{n-1} v_{n-1}^{0}(t, x)+O\left(\varepsilon^{n}\right), \quad \varepsilon \longrightarrow 0 .
\end{aligned}
$$

Here, the coefficient functions $v_{k}^{0}(t, x), k=1, \ldots, n-1$ are (formally) given as the solution of

$$
\begin{gathered}
\frac{\partial}{\partial t} v_{k}^{0}(t, x)+\mathscr{L}^{0} v_{k}^{0}(t, x)+g_{k}^{0}(t, x)=0, \\
(t, x) \in[0, T) \times D, \\
v_{k}^{0}(T, x)=0, \quad x \in D, \\
v_{k}^{0}(t, x)=0, \quad(t, x) \in[0, T] \times \partial D,
\end{gathered}
$$


where $g_{k}^{0}(t, x)$ is given inductively by

$$
g_{k}^{0}(t, x)=\widetilde{\mathscr{L}}_{k}^{0} u^{0}(t, x)+\sum_{l=1}^{k-1} \widetilde{\mathscr{L}}_{k-l}^{0} v_{l}^{0}(t, x)
$$

Here, $\widetilde{\mathscr{L}}_{k}^{0}$ is defined as follows:

$$
\begin{aligned}
\widetilde{\mathscr{L}}_{k}^{0}=\frac{1}{k !}\left\{\frac{1}{2} \sum_{i, j=1}^{d} \frac{\partial^{k} a^{i j}}{\partial \varepsilon^{k}}(x, 0) \frac{\partial^{2}}{\partial x^{i} \partial x^{j}}\right. \\
\left.+\sum_{i=1}^{d} \frac{\partial^{k} b^{i}}{\partial \varepsilon^{k}}(x, 0) \frac{\partial}{\partial x^{i}}-\frac{\partial^{k} c}{\partial \varepsilon^{k}}(x, 0)\right\} .
\end{aligned}
$$

Then, we can show the next result, whose proof is given in Appendix A.

Theorem 2. Assume $(A)-(H)$ which are stated in Section 2.2. Then, for each $k=1, \ldots, n-1, v_{k}^{0}$ is the classical solution of (18) and satisfies

$$
\left|v_{k}^{0}(t, x)\right| \leq C_{k}\left(1+|x|^{2 m_{k}}\right), \quad(t, x) \in[0, T] \times \mathbb{R}^{d}
$$

for some $C_{k}, m_{k}>0$.

Note that the uniqueness of the solutions of (18) follows from the same arguments as in the proof of Theorem 5.7.6 in [16]. That is, we obtain the next proposition.

Proposition 3. For any function $g$ which has a polynomial growth rate in $x$ uniformly in $t$, a classical solution of (18) is unique in the following sense: if $v$ and $w$ are classical solutions of $(18)$ and $|v(t, x)|+|w(t, x)| \leq C\left(1+|x|^{2 m}\right)$ for some $C, m>0$, then $v=w$.

Now, we are able to state our first main result on the asymptotic expansion. The proof is given in Appendix B.

Theorem 4. Assume $(A)-(H)$ which are given in Section 2.2. There are positive constants $C_{n}$ and $\widetilde{m}_{n}$ which are independent of $\varepsilon$ such that

$$
\begin{aligned}
& \left|u^{\varepsilon}(t, x)-\left(u^{0}(t, x)+\sum_{k=1}^{n-1} \varepsilon^{k} v_{k}^{0}(t, x)\right)\right| \\
& \quad \leq C_{n}\left(1+|x|^{2 \widetilde{m}_{n}}\right) \varepsilon^{n}, \quad(t, x) \in[0, T] \times \bar{D} .
\end{aligned}
$$

Next, we construct a semigroup corresponding to $\left(X_{t}^{0, x}\right)_{t}$ (i.e., $\left(X_{t}^{\varepsilon, x}\right)_{t}$ with $\varepsilon=0$ ) and $D$. Then, based on this semigroup we can obtain more explicit representation for the coefficient function $v_{k}^{0}(t, x)$ than the right hand side of (36) in Assumption (H), which will appear in Section 2.2.

Let $C_{b}^{0}(\bar{D})$ be the set of bounded continuous functions $f: \bar{D} \rightarrow \mathbb{R}$ such that $f(x)=0$ on $\partial D$. Obviously, $C_{b}^{0}(\bar{D})$ equipped with the supnorm becomes a Banach space. by

For $t \in[0, T]$ and $f \in C_{b}^{0}(\bar{D})$, we define $P_{t}^{D} f: \bar{D} \rightarrow \mathbb{R}$

$$
\begin{array}{r}
P_{t}^{D} f(x)=\mathrm{E}\left[\exp \left(-\int_{0}^{t} c\left(X_{v}^{0, x}, 0\right) d v\right)\right. \\
\left.\times f\left(X_{t}^{0, x}\right) 1_{\left\{\tau_{D}\left(X^{0, x}\right) \geq t\right\}}\right],
\end{array}
$$

where $c(x, 0)$ is nonnegative. We notice that $P_{t}^{D} f(x)$ is equal to $u^{0}(T-t, x)$ with the payoff function $f$. Then, we have the following result.

Proposition 5. Under the assumptions $(A)-(E)$ stated in Section 2.2, the mapping $P_{t}^{D}: C_{b}^{0}(\bar{D}) \rightarrow C_{b}^{0}(\bar{D})$ is well-defined and $\left(P_{t}^{D}\right)_{0 \leq t \leq T}$ is a contraction semigroup.

Proof. Let $f \in C_{b}^{0}(\bar{D})$. The relations $P_{0}^{D} f=f,\left.P_{t}^{D} f\right|_{\partial D}=0$, and $\sup _{\bar{D}}\left|P_{t}^{D} f\right| \leq \sup _{\bar{D}}|f|$ are obvious. The continuity of $P_{t}^{D} f$ is by Lemma 4.3 in [11]. The semigroup property is verified by a straightforward calculation.

Remark 6. Note that $\left(P_{t}^{D}\right)_{t}$ also has the semigroup property on the set $C_{p}^{0}(\bar{D})$ of continuous functions $f$, each of which has a polynomial growth rate and satisfies $f(x)=0$ on $\partial D$.

Finally, we show our second main result on the semigroup representation of the coefficient function $v_{k}^{0}$ in the expansion, whose proof is given in Appendix C.

Theorem 7. Under Assumptions $(A)-(H)$ given in Section 2.2, for each $k=1, \ldots, n-1$

$$
\begin{aligned}
& v_{k}^{0}(T-t, x) \\
& =\sum_{l=1}^{k} \sum_{\left(\beta^{i}\right)_{i=1}^{l} \subset \mathbb{N}^{l}, \sum_{i} \beta^{i}=k} \int_{0}^{t} \int_{0}^{t_{1}} \cdots \int_{0}^{t_{l-1}} P_{t-t_{1}}^{D} \widetilde{\mathscr{L}}_{\beta^{1}}^{0} P_{t_{1}-t_{2}}^{D} \\
& \times \widetilde{\mathscr{L}}_{\beta^{2}}^{0} \cdots P_{t_{l-1}-t_{l}}^{D} \\
& \times \widetilde{\mathscr{L}}_{\beta^{l}}^{0} P_{t_{l}}^{D} f(x) d t_{l} \cdots d t_{1} \text {. }
\end{aligned}
$$

2.2. Assumptions. This subsection introduces a series of the assumptions necessary for our main results stated in the previous subsection. Particularly, the assumptions (A)-(E) are relevant for Theorem 1 , that is the existence and uniqueness result of the PDE (14), while $(\mathrm{F})-(\mathrm{H})$ are additional assumptions necessary for the asymptotic expansion results, that is Theorems 2-7.

(A) There is a positive constant $A_{1}$ such that

$$
\begin{array}{r}
\left|\sigma^{i j}(x, \varepsilon)\right|^{2}+\left|b^{i}(x, \varepsilon)\right|^{2} \leq A_{1}\left(1+|x|^{2}\right), \\
x \in \mathbb{R}^{d}, \quad \varepsilon \in I, \quad i, j=1, \ldots, d .
\end{array}
$$


Moreover, for each $\varepsilon \in I$ it holds that $\sigma^{i j}(\cdot, \varepsilon), b^{i}(\cdot, \varepsilon) \in$ Lip for $i, j=1, \ldots, d$, where Lip is the set of locally Lipschitz continuous functions defined on $\mathbb{R}^{d}$ :

Lip $=\left\{f \in C\left(\mathbb{R}^{d} ; \mathbb{R}\right) ;\right.$ for any compact set $K \subset \mathbb{R}^{d}$,

$\exists C_{K}>0$ such that $|f(x)-f(y)| \leq C_{K}|x-y|$,

$x, y \in K\}$.

Remark 8. Note that under (A), the existence and uniqueness of a solution of (10) are guaranteed on any filtered probability space equipped with a standard $m$-dimensional Brownian motion, and Corollary 2.5.12 in [17] and Lemma 3.2.6 in [18] imply

$$
\begin{aligned}
\mathrm{E}\left[\sup _{0 \leq r \leq t}\left|X_{r}^{\varepsilon, x}-x\right|^{2 l}\right] & \leq C_{l} t^{l} e^{C_{l} t}\left(1+|x|^{2 l}\right), \\
(t, x) & \in[0, T] \times \mathbb{R}^{d}, \quad l \in \mathbb{N}
\end{aligned}
$$

for some $C_{l}>0$, which depends only on $l, A_{1}$, and $C_{K}$. Moreover, $\left(X_{r}^{x}\right)_{r}$ has the strong Markov property.

(B) The function $f(x)$ is continuous on $\bar{D}$ and there are $C_{f}>0$ and $m \in \mathbb{N}$ such that $|f(x)| \leq C_{f}\left(1+|x|^{2 m}\right)$, $x \in \mathbb{R}^{d}$. Moreover, $f(x)=0$ on $\mathbb{R}^{d} \backslash D$.

Remark 9. The assumption (B) which corresponds to $\mathbf{H 2 ( 2 )}$ in [11] guarantees the continuity of a solution of (14) (if it exists) on the so-called parabolic boundary $\Sigma=\partial D \times[0, T) \cup$ $\bar{D} \times\{T\}$. For the details, see page 8 in [11].

(C) $c(x, \varepsilon)$ is nonnegative (i.e., $c(x, \varepsilon) \geq 0)$. Moreover, for each $\varepsilon \in I$, it holds that $c(\cdot, \varepsilon) \in$ Lip.

(D) The boundary $\partial D$ has the outside strong sphere property; that is, for each $x \in \partial D$ there is a closed ball $E$ such that $E \cap D=\emptyset$ and $E \cap \bar{D}=\{x\}$.

Remark 10. The assumption $(D)$ provides the regularity of each point in $\partial D$. (compared to [8]) Also, [11] points out that $(D)$ with the ellipticity of the matrix $\left(a^{i j}(x, \varepsilon)\right)_{i j}$ in (E) below gives

$$
P\left(\tau_{D}\left(X^{\epsilon, x}\right)=\tau_{\bar{D}}\left(X^{\epsilon, x}\right)\right)=1 .
$$

(E) The matrix $\left(a^{i j}(x, \varepsilon)\right)_{i j}$ is locally elliptic in the sense that for each $\varepsilon \in I$ and compact set $K \subset \mathbb{R}^{d}$ there is a positive number $\mu_{\varepsilon, K}$ such that $\sum_{i, j=1}^{d} a^{i j}(x, \varepsilon) \xi^{i} \xi^{j} \geq$ $\mu_{\varepsilon, K}|\xi|^{2}$ for any $x \in K$ and $\xi \in \mathbb{R}^{d}$.

Remark 11. Note that although the condition (E) (local ellipticity) is necessary for the existence of classical solution of our PDE (see Remark 2.2 in [11]), the assumption can be removed through consideration of viscosity solutions rather than classical solutions by applying Theorem 8.2 in [19] and
Theorems 4.4.3 and 7.7.2 in [18]. Note that we need the additional assumption such that $I \subset[0, \infty)$ by technical reason in this case.

To study the asymptotic expansion, we put the following assumptions in addition to (A)-(E). Firstly, by the next condition we can properly define $\widetilde{\mathscr{L}}_{k}^{0}, k \in \mathbb{N}$ in (20) above.

(F) Let $n \in \mathbb{N}$. The functions $a^{i j}(x, \varepsilon), b^{i}(x, \varepsilon)$, and $c(x, \varepsilon)$ are $n$-times continuously differentiable in $\varepsilon$. Furthermore, each of derivatives $\partial^{k} a^{i j} / \partial \varepsilon^{k}, \partial^{k} b^{i} / \partial \varepsilon^{k}$, $\partial^{k} c / \partial \varepsilon^{k}, k=1, \ldots, n-1$, has a polynomial growth rate in $x \in \mathbb{R}^{d}$ uniformly in $\varepsilon \in I$.

To state the existence of the functions $v_{k}^{0}(t, x)$ in the asymptotic expansion (17), we first prepare the following set.

Definition 12. The set $\mathscr{H}^{m, p}$ of $g \in C([0, T) \times \bar{D})$ is defined to satisfy the following condition. There is a $M^{g} \in C([0, T)) \cap$ $L^{p}([0, T), d t)$ such that

$$
|g(t, x)| \leq M^{g}(t)\left(1+|x|^{2 m}\right), \quad t \in[0, T), x \in \bar{D} .
$$

Given this definition of the set $\mathscr{H}^{m, p}$, we put the next condition on $u^{0}$.

(G) $u^{0} \in \mathscr{G}^{m}$, where

$$
\begin{aligned}
\mathscr{G}^{m}=\left\{u \in C^{1,2}([0, T) \times D) \cap C([0, T] \times \bar{D}) ;\right. \\
\left.\quad \frac{\partial u}{\partial x^{i}} \in \mathscr{H}^{m, 2}, \frac{\partial^{2} u}{\partial x^{i} \partial x^{j}} \in \mathscr{H}^{m, 1}, i, j=1, \ldots, d\right\} .
\end{aligned}
$$

Now we examine the conditions necessary for the classical solution to the PDE (18).

Firstly, Let us start with the case of $k=1$. By the assumption (G), we have $g_{1}^{0} \in \mathscr{H}^{m, 1}$ for some $m \in \mathbb{N}$ by the definition of $g_{k}^{0}$ with $k=1$ in (19). Thus we can define

$$
\begin{aligned}
& v_{1}^{0}(t, x) \\
& =\mathrm{E}\left[\int_{0}^{(T-t) \wedge \tau_{D}\left(X^{0, x}\right)} \exp \left(-\int_{0}^{r} c\left(X_{v}^{0, x}, 0\right) d v\right)\right. \\
& \left.\quad \times g_{1}^{0}\left(t+r, X_{r}^{0, x}\right) d r\right] .
\end{aligned}
$$

Therefore, if we assume that $v_{1}^{0} \in C^{1,2}([0, T) \times D)$ we can show that $v_{1}^{0}$ is the solution of (18) with $k=1$; that is, we can confirm that

$$
\frac{\partial}{\partial t} v_{1}^{0}(t, x)+\mathscr{L}^{0} v_{1}^{0}(t, x)+g_{1}^{0}(t, x)=0 .
$$

Note that the relations $v_{1}^{0}(T, \cdot)=0$ and $v_{1}^{0}=0$ on $[0, T] \times \partial D$ are obvious. 
Next, let us give some comments on the smoothness of $v_{1}^{0}$. In many cases as in the Black-Scholes model (see (49) in Section 3) we can rewrite (31) as

$$
v_{1}^{0}(t, x)=\int_{0}^{T-t} \int_{D} g_{1}^{0}(t+r, y) p(r, x, y) d y d r
$$

for some $p(r, x, y)$. Thus, if $p$ has a "good" smoothness property, the smoothness of $v_{1}^{0}$ also holds such as

$$
\begin{aligned}
\frac{\partial}{\partial t} v_{1}^{0}(t, x)= & -\lim _{s \rightarrow T} \int_{D} g_{1}^{0}(s, y) p(s-t, x, y) d y \\
& +\int_{0}^{t} \int_{D} \frac{\partial}{\partial t} g_{1}^{0}(t+r, y) p(r, x, y) d r
\end{aligned}
$$

if the limit in the right hand side exists, and

$$
\begin{gathered}
\frac{\partial}{\partial x^{i}} v_{1}^{0}(t, x)=\int_{0}^{t} \int_{D} g_{1}^{0}(t+r, y) \frac{\partial}{\partial x^{i}} p(r, x, y) d r \\
\frac{\partial^{2}}{\partial x^{i} \partial x^{j}} v_{1}^{0}(t, x)=\int_{0}^{t} \int_{D} g_{1}^{0}(t+r, y) \frac{\partial^{2}}{\partial x^{i} \partial x^{j}} p(r, x, y) d r .
\end{gathered}
$$

Moreover, if $v_{1}^{0}$ is in $\mathscr{G}^{m_{1}}$ for some $m_{1} \in \mathbb{N}$, we also have $g_{2}^{0} \in \mathscr{H}^{\widetilde{m}_{1}, 1}$ for some $\widetilde{m}_{1} \in \mathbb{N}$ by the definition of $g_{k}^{0}$ with $k=2$ in (19). Then, we can define $v_{2}^{0}$ similarly as $v_{1}^{0}$. Furthermore, under some suitable smoothness conditions for $v_{2}^{0}$, which may be given by the smoothness property of $p(r, x, y)$, we are able to show that $v_{2}^{0}$ is the classical solution of (18) with $k=2$. tion.

Thus, the observation above leads us to our final assump-

(H) It holds that $v_{k}^{0} \in \mathscr{G}^{m_{n}}, k=1, \ldots, n-1$ for some $m_{n} \in$ $\mathbb{N}$, where

$$
\begin{gathered}
v_{k}^{0}(t, x)=\mathrm{E}\left[\int_{0}^{(T-t) \wedge \tau_{D}\left(X^{0, x}\right)} \exp \left(-\int_{0}^{r} c\left(X_{v}^{0, x}, 0\right) d v\right)\right. \\
\left.\times g_{k}^{0}\left(t+r, X_{r}^{0, x}\right) d r\right]
\end{gathered}
$$

\section{Application to Barrier Option Pricing in Stochastic Volatility Environment}

This section demonstrates the effectiveness of our method in stochastic volatility environment. Section 3.1 derives concrete approximation formulas, and Section 3.2 shows numerical examples.

3.1. Approximation of Barrier Option Prices in a Stochastic Volatility Model. We consider the following stochastic volatility model under the risk-neutral probability measure:

$$
\begin{gathered}
d S_{t}^{\varepsilon}=(c-q) S_{t}^{\varepsilon} d t+\sigma_{t}^{\varepsilon} S_{t}^{\varepsilon} d B_{t}^{1}, \quad S_{0}^{\varepsilon}=S, \\
d \sigma_{t}^{\varepsilon}=\varepsilon \lambda\left(\theta-\sigma_{t}^{\varepsilon}\right) d t+\varepsilon \nu \sigma_{t}^{\varepsilon}\left(\rho d B_{t}^{1}+\sqrt{1-\rho^{2}} d B_{t}^{2}\right), \\
\sigma_{0}^{\varepsilon}=\sigma,
\end{gathered}
$$

where $c, q>0, \varepsilon \in[0,1), \lambda, \theta, \nu>0$, and $\rho \in[-1,1]$ and $B=\left(B^{1}, B^{2}\right)$ is a two dimensional Brownian motion. Here $c$ and $q$ represent a domestic interest rate and a foreign interest rate, respectively, when we consider the currency options.

Clearly, applying Itô's formula, we have its logarithmic process:

$$
\begin{gathered}
d X_{t}^{\varepsilon}=\left(c-q-\frac{1}{2}\left(\sigma_{t}^{\varepsilon}\right)^{2}\right) d t+\sigma_{t}^{\varepsilon} d B_{t}^{1}, \\
X_{0}^{\varepsilon}=x=\log S, \\
d \sigma_{t}^{\varepsilon}=\varepsilon \lambda\left(\theta-\sigma_{t}^{\varepsilon}\right) d t+\varepsilon \nu \sigma_{t}^{\varepsilon}\left(\rho d B_{t}^{1}+\sqrt{1-\rho^{2}} d B_{t}^{2}\right), \\
\sigma_{0}^{\varepsilon}=\sigma .
\end{gathered}
$$

Also, its generator is expressed as

$$
\begin{aligned}
\mathscr{L}^{\varepsilon}= & \left(c-q-\frac{1}{2} \sigma^{2}\right) \frac{\partial}{\partial x}+\frac{1}{2} \sigma^{2} \frac{\partial^{2}}{\partial x^{2}} \\
& +\varepsilon \rho \nu \sigma^{2} \frac{\partial^{2}}{\partial x \partial \sigma}+\varepsilon \lambda(\theta-\sigma) \frac{\partial}{\partial \sigma} \\
& +\varepsilon^{2} \frac{1}{2} \nu^{2} \sigma^{2} \frac{\partial^{2}}{\partial \sigma^{2}}-c .
\end{aligned}
$$

In this case, $\widetilde{\mathscr{L}}_{1}^{0}$ which is defined by (20) with $k=1$ is given as

$$
\widetilde{\mathscr{L}}_{1}^{0}=\rho \nu \sigma^{2} \frac{\partial^{2}}{\partial x \partial \sigma}+\lambda(\theta-\sigma) \frac{\partial}{\partial \sigma} .
$$

We will apply the asymptotic expansion in the previous section to (38) and give an approximation formula for a barrier option price, which is given under a risk-neutral probability measure as

$$
C_{\text {Barrier }}^{\mathrm{SV}, \varepsilon}\left(T-t, e^{x}\right)=\mathrm{E}\left[e^{-c(T-t)} f\left(S_{T-t}^{\varepsilon, e^{x}}\right) 1_{\left\{\tau_{(L, \infty)}\left(S^{\varepsilon, e^{x}}\right)>T-t\right\}}\right],
$$

where $f$ stands for a payoff function, $L(<S)$ is a barrier price and the expectation is taken with respect to the risk-neutral probability measure. PDE:

Then, $u^{\varepsilon}(t, x)=C_{\mathrm{Barrier}}^{\mathrm{SV}, \varepsilon}\left(T-t, e^{x}\right)$ satisfies the following

$$
\begin{gathered}
\left(\frac{\partial}{\partial t}+\mathscr{L}^{\varepsilon}\right) u^{\varepsilon}(t, x)=0, \quad(t, x) \in(0, T] \times D, \\
u^{\varepsilon}(T, x)=\bar{f}(x), \quad x \in \bar{D}, \\
u^{\varepsilon}(t, l)=0, \quad t \in[0, T],
\end{gathered}
$$

where $\bar{f}(x)=\max \left\{e^{x}-K, 0\right\}, D=(l, \infty)$, and $l=\log L$. We obtain the 0 th order $u^{0}$ as

$$
u^{0}(t, x)=P_{T-t}^{D} \bar{f}(x)=\mathrm{E}\left[e^{-c(T-t)} \bar{f}\left(X_{T-t}^{x, 0}\right) 1_{\left\{\tau_{D}\left(X^{0, x}\right)>T-t\right\}}\right] .
$$


Remark 13. $u^{0}$ satisfies PDE (42) with $\varepsilon=0$. Although the condition (E) in Section 2 does not seem to be satisfied in this case, the volatility process $\left(\sigma_{t}^{\varepsilon}\right)_{t}$ becomes a constant $\sigma>0$, and so (38) is reduced to a one-dimensional SDE. Then, (42) with $\varepsilon=0$ becomes a nondegenerating PDE with fixed $\sigma$. Therefore, we need not take care of the lack of the condition (E) in this example.

Setting $\alpha=c-q$, we note that $P_{T-t}^{D} \bar{f}(x)=C_{\text {Barrier }}^{\mathrm{BS}}(T-$ $\left.t, e^{x}, \alpha, \sigma, L\right)$ is the price of the down-and-out barrier call option under the Black-Scholes model:

$$
\begin{aligned}
& C_{\text {Barrier }}^{\mathrm{BS}}\left(T-t, e^{x}, \alpha, \sigma, L\right) \\
& =C^{\mathrm{BS}}\left(T-t, e^{x}, \alpha, \sigma\right) \\
& \quad-\left(\frac{e^{x}}{L}\right)^{1-2 \alpha / \sigma^{2}} C^{\mathrm{BS}}\left(T-t, \frac{L^{2}}{e^{x}}, \alpha, \sigma\right) .
\end{aligned}
$$

Here, we recall that the price of the plain vanilla option under the Black-Scholes model is given as

$$
\begin{aligned}
C^{\mathrm{BS}}\left(T-t, e^{x}, \alpha, \sigma\right)= & e^{-q(T-t)} e^{x} N\left(d_{1}(T-t, x, \alpha)\right) \\
& -e^{-c(T-t)} K N\left(d_{2}(T-t, x, \alpha)\right),
\end{aligned}
$$

where

$$
\begin{gathered}
d_{1}(t, x, \alpha)=\frac{x-\log K+\alpha t}{\sigma \sqrt{t}}+\frac{1}{2} \sigma \sqrt{t}, \\
d_{2}(t, x, \alpha)=d_{1}(t, x, \alpha)-\sigma \sqrt{t} \\
N(x)=\int_{-\infty}^{x} n(y) d y, \\
n(y)=\frac{1}{\sqrt{2 \pi}} e^{-y^{2} / 2} .
\end{gathered}
$$

Note also that

$$
\begin{aligned}
& P\left(\tau_{D}\left(X^{0, x}\right) \geq t \mid X_{t}^{0, x}\right) \\
& \quad=1-\exp \left(-\frac{2(x-l)\left(X_{t}^{0, x}-l\right)}{\sigma^{2} t}\right) \text { on }\left\{X_{t}^{0, x}>l\right\} .
\end{aligned}
$$

Therefore, for $g \in C_{p}^{0}(\bar{D})$ we have

$$
\begin{aligned}
P_{t}^{D} g(x) & =\mathrm{E}\left[P\left(\tau_{D}\left(X^{0, x}\right) \geq t \mid X_{t}^{x}\right) e^{-c t} g\left(X_{t}^{0, x}\right) 1_{\left\{X_{t}^{0, x}>l\right\}}\right] \\
& =\int_{l}^{\infty} e^{-c t} g(y) p(t, x, y) d y,
\end{aligned}
$$

where

$$
\begin{aligned}
p(t, x, y)= & \frac{1}{\sqrt{2 \pi \sigma^{2} t}}\left(1-e^{-2(x-l)(y-l) / \sigma^{2} t}\right) \\
& \times e^{-(y-x-\mu t)^{2} / 2 \sigma^{2} t}, \\
\mu=\alpha & -\frac{\sigma^{2}}{2}=\left(c-q-\frac{\sigma^{2}}{2}\right) .
\end{aligned}
$$

Then, we show the following main result in this section.

Theorem 14. We obtain an approximation formula for the down-and-out barrier call option under the stochastic volatility model (37):

$$
\begin{aligned}
C_{\text {Barrier }}^{S V, \varepsilon}\left(T, e^{x}\right)= & C_{\text {Barrier }}^{B S}\left(T, e^{x}, \alpha, \sigma, L\right)+\varepsilon v_{1}^{0}(0, x) \\
& +O\left(\varepsilon^{2}\right),
\end{aligned}
$$

where

$$
v_{1}^{0}(0, x)
$$

$$
=e^{-c T} \int_{0}^{T} \int_{l}^{\infty} \frac{1}{\sqrt{2 \pi \sigma^{2} s}}\left(1-e^{-2(x-l)(y-l) / \sigma^{2} s}\right)
$$

$$
\times e^{-\left(y-x-\left(\alpha-(1 / 2) \sigma^{2}\right) s\right)^{2} / 2 \sigma^{2} s} \vartheta(s, y) d y d s,
$$

$$
\begin{aligned}
& \vartheta(t, x)=e^{\alpha(T-t)} \rho \nu \sigma e^{x} n\left(d_{1}(T-t, x, \alpha)\right) \\
& \times\left(-d_{2}(T-t, x, \alpha)\right) \\
& +2 e^{\alpha(T-t)} \rho \nu \alpha\left(\frac{e^{x}}{L}\right)^{-2 \alpha / \sigma^{2}} \\
& \times \operatorname{Ln}\left(c_{1}(T-t, x, \alpha)\right) \sqrt{T-t} \\
& -e^{\alpha(T-t)} \rho \nu \sigma\left(\frac{e^{x}}{L}\right)^{-2 \alpha / \sigma^{2}} \\
& \times \operatorname{Ln}\left(c_{1}(T-t, x, \alpha)\right) c_{1}(T-t, x, \alpha) \\
& -e^{c(T-t)} \rho \nu \frac{4 \alpha}{\sigma}\left(\frac{e^{x}}{L}\right)^{1-2 \alpha / \sigma^{2}} \\
& \times\left\{C^{B S}\left(T-t, \frac{L^{2}}{e^{x}}, \alpha, \sigma\right)\right. \\
& \times\left\{1+(x-\log L)\left(1-\frac{2 \alpha}{\sigma^{2}}\right)\right\} \\
& \left.-(x-\log L) e^{-q(T-t)} \frac{L^{2}}{e^{x}} N\left(c_{1}(T-t, x, \alpha)\right)\right\}
\end{aligned}
$$




$$
\begin{aligned}
& +\lambda(\theta-\sigma) e^{\alpha(T-t)} e^{x} n\left(d_{1}(T-t, x, \alpha)\right) \sqrt{T-t} \\
& -\lambda(\theta-\sigma)\left(\frac{e^{x}}{L}\right)^{-2 \alpha / \sigma^{2}} e^{\alpha(T-t)} \\
& \times \operatorname{Ln}\left(c_{1}(T-t, x, \alpha)\right) \sqrt{T-t} \\
& -e^{c(T-t)} \lambda(\theta-\sigma) \frac{4 \alpha}{\sigma^{3}}\left(\log \frac{e^{x}}{L}\right)\left(\frac{e^{x}}{L}\right)^{1-2 \alpha / \sigma^{2}} \\
& \times C^{B S}\left(T-t, \frac{L^{2}}{e^{x}}, \alpha, \sigma\right), \\
& c_{1}(t, x, \alpha)=\frac{2 l-x-\log K+\alpha t}{\sigma \sqrt{t}}+\frac{1}{2} \sigma \sqrt{t} .
\end{aligned}
$$

Proof. Firstly, note that when $k=1$ in Theorem 7, we have

$$
v_{1}^{0}(T-t, x)=\int_{0}^{t} P_{t-r}^{D} \widetilde{\mathscr{L}}_{1}^{0} P_{r}^{D} f(x) d r .
$$

Thus, we see the expansion

$$
\begin{aligned}
C_{\text {Barrier }}^{\mathrm{SV}, \varepsilon}\left(T-t, e^{x}\right)= & C_{\text {Barrier }}^{\mathrm{BS}}\left(T-t, e^{x}, \alpha, \sigma, L\right) \\
& +\varepsilon \int_{0}^{T-t} P_{s}^{D} \widetilde{\mathscr{L}}_{1}^{0} P_{T-t-s}^{D} \bar{f}(x) d s+O\left(\varepsilon^{2}\right) .
\end{aligned}
$$

The first-order approximation term $v_{1}^{0}(t, x)=$ $\int_{0}^{T-t} P_{s}^{D} \widetilde{\mathscr{L}}_{1}^{0} P_{T-t-s}^{D} \bar{f}(x) d s$ is given by

$$
\begin{aligned}
v_{1}^{0}(t, x) & =\int_{0}^{T-t} e^{-c s} \bar{P}_{s}^{D} \widetilde{\mathscr{L}}_{1}^{0} e^{-c(T-t-s)} \bar{P}_{T-t-s}^{D} \bar{f}(x) d s \\
& =e^{-c(T-t)} \int_{0}^{T-t} \bar{P}_{s}^{D} \widetilde{\mathscr{L}}_{1}^{0} \bar{P}_{T-t-s}^{D} \bar{f}(x) d s
\end{aligned}
$$

where $\bar{P}_{t}^{D}$ is defined by

$$
\begin{aligned}
\bar{P}_{t}^{D} \bar{f}(x)=\int_{l}^{\infty} & \frac{1}{\sqrt{2 \pi \sigma^{2} s}}\left(1-e^{-2(x-l)(y-l) / \sigma^{2} s}\right) \\
& \times e^{-\left(y-x-\left(\alpha-(1 / 2) \sigma^{2}\right) s\right)^{2} / 2 \sigma^{2} s} \bar{f}(y) d y .
\end{aligned}
$$

Define $\vartheta(t, x)$ as

$$
\begin{aligned}
\vartheta(t, x)= & \widetilde{\mathscr{L}}_{1}^{0} \bar{P}_{T-t}^{D} f\left(e^{x}\right) \\
= & e^{c(T-t)} \rho \nu \sigma^{2} \frac{\partial^{2}}{\partial x \partial \sigma} C_{\text {Barrier }}^{\mathrm{BS}}\left(T-t, e^{x}, \alpha, \sigma, L\right) \\
& +e^{c(T-t)} \lambda(\theta-\sigma) \frac{\partial}{\partial \sigma} C_{\text {Barrier }}^{\mathrm{BS}}\left(T-t, e^{x}, \alpha, \sigma, L\right) .
\end{aligned}
$$

A straightforward calculation shows that the above function agrees with the right-hand side of (52). Then we get the assertion.
Remark that through numerical integrations with respect to time $s$ and space $y$ in (51), we easily obtain the first order approximation of the down-and-out option prices.

Next, as a special case of (37) we consider the following stochastic volatility model with no drifts:

$$
\begin{gathered}
d S_{t}^{\varepsilon}=\sigma_{t}^{\varepsilon} S_{t}^{\varepsilon} d B_{t}^{1}, \\
S_{0}^{\varepsilon}=S>0, \\
d \sigma_{t}^{\varepsilon}=\varepsilon v \sigma_{t}^{\varepsilon}\left(\rho d B_{t}^{1}+\sqrt{1-\rho^{2}} d B_{t}^{2}\right), \\
\sigma_{0}^{\varepsilon}=\sigma>0,
\end{gathered}
$$

where $\varepsilon \in[0,1), \rho \in[-1,1]$ and $B=\left(B^{1}, B^{2}\right)$ is a twodimensional Brownian motion. In this case, we can provide a simpler approximation formula than in Theorem 14 .

By Itô's formula, the following logarithmic model is obtained:

$$
\begin{gathered}
d X_{t}^{\varepsilon}=-\frac{1}{2}\left(\sigma_{t}^{\varepsilon}\right)^{2} d t+\sigma_{t}^{\varepsilon} d B_{t}^{1}, \\
X_{0}^{\varepsilon}=x=\log S, \\
d \sigma_{t}^{\varepsilon}=\varepsilon v \sigma_{t}^{\varepsilon}\left(\rho d B_{t}^{1}+\sqrt{1-\rho^{2}} d B_{t}^{2}\right), \\
\sigma_{0}^{\varepsilon}=\sigma .
\end{gathered}
$$

Again, the barrier option price is given by

$$
C_{\text {Barrier }}^{\mathrm{SV}, \varepsilon}\left(T, e^{x}\right)=\mathrm{E}\left[f\left(S_{T}^{\varepsilon}\right) 1_{\left\{\min _{0 \leq u \leq T} S_{u}^{\varepsilon}>L\right\}}\right] \text {, }
$$

where $f$ stands for a payoff function and $L(<S)$ is a barrier price.

The differential operators $\mathscr{L}^{\varepsilon}, \widetilde{\mathscr{L}}_{1}^{0}$ and the PDE are same as (39)-(42) with $c=q=0$ and $\lambda=0$. Also, the barrier option price in the Black-Scholes model coincides with (44) with no drift; that is,

$$
C_{\text {Barrier }}^{\mathrm{BS}}(T, S)=C^{\mathrm{BS}}(T, S)-\left(\frac{S}{L}\right) C^{\mathrm{BS}}\left(T, \frac{L^{2}}{S}\right),
$$

where $C^{\mathrm{BS}}(T, S)$ is the driftless Black-Scholes formula of the European call option given by

$$
C^{\mathrm{BS}}(T, S)=S N\left(d_{1}(T, \log S)\right)-K N\left(d_{2}(T, \log S)\right)
$$

with

$$
\begin{gathered}
d_{1}(t, x)=d_{1}(t, x, 0)=\frac{x-\log K+\sigma^{2} t / 2}{\sigma \sqrt{t}}, \\
d_{2}(t, x)=d_{2}(t, x, 0)=d_{1}(t, x)-\sigma \sqrt{t} .
\end{gathered}
$$

Then, we reach the following expansion formula which only needs 1-dimensional numerical integration. 
Theorem 15. $C_{\text {Barrier }}^{S V, \varepsilon}\left(T, e^{x}\right)=C_{\text {Barrier }}^{B S}\left(T, e^{x}\right)+\varepsilon v_{1}^{0}(0, x)+$ $O\left(\varepsilon^{2}\right)$, where

$$
\begin{aligned}
& v_{1}^{0}(0, x)=- \frac{1}{2} T \nu \rho \sigma\left\{e^{x} n\left(d_{1}(T, x)\right) d_{2}(T, x)\right. \\
&\left.+\operatorname{Ln}\left(c_{1}(T, x)\right) c_{1}(T, x)\right\} \\
&+ \frac{\nu \rho L(x-l) \log (L / K)}{2 \pi \sigma} \\
& \times \int_{0}^{T} \frac{(T-s)^{1 / 2}}{s^{3 / 2}} \\
& \times \exp \left(-\frac{c_{2}(T-s, L / K)+c_{2}\left(s, L / e^{x}\right)}{2}\right) d s, \\
& c_{1}(t, x)= \frac{\log \left(L^{2} / e^{x} K\right)+\sigma^{2} t / 2}{\sigma \sqrt{t}}, \\
& c_{2}(t, y)=\left(\frac{\log y+\sigma^{2} t / 2}{\sigma \sqrt{t}}\right)^{2} .
\end{aligned}
$$

Proof. See Appendix D.

3.2. Numerical Example. Finally, applying our approximation formulas in Theorems 14 and 15, we present numerical experiments for European down-and-out barrier call prices. First, let us denote $u^{0}=C_{\text {Barrier }}^{\mathrm{BS}}(T, S)$ and $v_{1}^{0}=v_{1}^{0}(0, \log S)$. Then, we see

$$
C_{\text {Barrier }}^{\mathrm{SV}, \mathcal{E}}(T, S) \simeq u^{0}+\varepsilon v_{1}^{0} .
$$

In the following we report the results of the numerical experiments, where the numbers in the parentheses show the error rates (\%) relative to the benchmark prices of $C_{\text {Barrier }}^{S V, \varepsilon}(T, S)$; they are computed by Monte-Carlo simulations with 100,000 time steps $\left(n=10^{5}\right)$ and 1,000,000 trials. We note that in our experiments the standard deviations of the benchmark Monte-Carlo simulations are calculated as at most 0.006 with the order of discretization error being 0.002 $\left(\approx \sqrt{T / n}=\sqrt{0.5 / 10^{5}}\right.$ as stated in Introduction $)$.

We check the accuracy of our approximations by changing the model parameters. Cases 1-6 show the results for the stochastic volatility model with drifts of the underlying price process or/and the volatility process (37), while Case 7 shows the result for the stochastic volatility model with no drifts (59) (see Tables 1, 2, 3, 4, 5, 6, and 7). There, we apply the formula in Theorem 14 to Cases 1-6 and the formula in Theorem 15 to Case 7, respectively.

In all the cases, we set the initial asset price $S=100$, the initial volatility $\sigma=0.15$, the time to maturity $T=0.5$, the lower barrier $L=95$, and strike prices $K=100,102,105$. The other parameters $(c, q, \varepsilon \nu, \rho, \varepsilon \lambda, \theta)$ are listed in the caption of each table.

Apparently, our approximation formula $u^{0}+\varepsilon v_{1}^{0}$ improves the accuracy against $C_{\mathrm{Barrier}}^{\mathrm{SV}, \mathcal{E}}(T, S)$, and it is observed that $\varepsilon v_{1}^{0}$ accurately compensates for the difference between
TABle 1: Case $1(c=0.01, q=0.0, \varepsilon v=0.2, \rho=-0.5, \varepsilon \lambda=0.0$, $\theta=0.0)$.

\begin{tabular}{lccc}
\hline Strike & Benchmark & $\begin{array}{c}\text { Our approximation } \\
\left(u^{0}+\varepsilon v_{1}^{0}\right)\end{array}$ & $\begin{array}{c}\text { Barrier } \\
\text { Black-Scholes }\left(u^{0}\right)\end{array}$ \\
\hline 100 & 3.468 & $3.466(-0.05 \%)$ & $3.495(0.80 \%)$ \\
102 & 2.822 & $2.822(0.00 \%)$ & $2.866(1.57 \%)$ \\
105 & 1.986 & $1.986(0.01 \%)$ & $2.052(3.36 \%)$ \\
\hline
\end{tabular}

TABLE 2: Case $2(c=0.01, q=0.0, \varepsilon v=0.35, \rho=-0.7, \varepsilon \lambda=0.0$, $\theta=0.0)$.

\begin{tabular}{lccc}
\hline Strike & Benchmark & $\begin{array}{c}\text { Our approximation } \\
\left(u^{0}+\varepsilon v_{1}^{0}\right)\end{array}$ & $\begin{array}{c}\text { Barrier } \\
\text { Black-Scholes }\left(u^{0}\right)\end{array}$ \\
\hline 100 & 3.421 & $3.423(0.07 \%)$ & $3.495(2.18 \%)$ \\
102 & 2.753 & $2.757(0.18 \%)$ & $2.866(4.13 \%)$ \\
105 & 1.885 & $1.890(0.23 \%)$ & $2.052(8.88 \%)$ \\
\hline
\end{tabular}

TABle 3: Case $3(c=0.05, q=0.0, \varepsilon \nu=0.35, \rho=-0.7, \varepsilon \lambda=0.0$, $\theta=0.0)$.

\begin{tabular}{lccc}
\hline Strike & Benchmark & $\begin{array}{c}\text { Our approximation } \\
\left(u^{0}+\varepsilon v_{1}^{0}\right)\end{array}$ & $\begin{array}{c}\text { Barrier } \\
\text { Black-Scholes }\left(u^{0}\right)\end{array}$ \\
\hline 100 & 4.352 & $4.349(-0.07 \%)$ & $4.399(1.06 \%)$ \\
102 & 3.585 & $3.586(0.02 \%)$ & $3.665(2.24 \%)$ \\
105 & 2.560 & $2.563(0.11 \%)$ & $2.696(5.31 \%)$ \\
\hline
\end{tabular}

TABLe 4: Case $4(c=0.05, q=0.1, \varepsilon v=0.2, \rho=-0.5, \varepsilon \lambda=0.0$, $\theta=0.0)$.

\begin{tabular}{lccc}
\hline Strike & Benchmark & $\begin{array}{c}\text { Our approximation } \\
\left(u^{0}+\varepsilon v_{1}^{0}\right)\end{array}$ & $\begin{array}{c}\text { Barrier } \\
\text { Black-Scholes }\left(u^{0}\right)\end{array}$ \\
\hline 100 & 2.231 & $2.224(-0.31 \%)$ & $2.268(1.64 \%)$ \\
102 & 1.758 & $1.754(-0.27 \%)$ & $1.812(3.02 \%)$ \\
105 & 1.172 & $1.168(-0.31 \%)$ & $1.243(6.05 \%)$ \\
\hline
\end{tabular}

TABle 5: Case $5(c=0.01, q=0.0, \varepsilon \nu=0.2, \rho=-0.5, \varepsilon \lambda=0.2$, $\theta=0.25)$.

\begin{tabular}{lccc}
\hline Strike & Benchmark & $\begin{array}{c}\text { Our approximation } \\
\left(u^{0}+\varepsilon v_{1}^{0}\right)\end{array}$ & $\begin{array}{c}\text { Barrier } \\
\text { Black-Scholes }\left(u^{0}\right)\end{array}$ \\
\hline 100 & 3.523 & $3.517(-0.16 \%)$ & $3.495(-0.77 \%)$ \\
102 & 2.891 & $2.888(-0.09 \%)$ & $2.866(-0.85 \%)$ \\
105 & 2.066 & $2.065(-0.06 \%)$ & $2.052(-0.64 \%)$ \\
\hline
\end{tabular}

TABLE 6: Case $6(c=0.01, q=0.0, \varepsilon v=0.2, \rho=-0.5, \varepsilon \lambda=0.5$, $\theta=0.25)$.

\begin{tabular}{lccc}
\hline Strike & Benchmark & $\begin{array}{c}\text { Our approximation } \\
\left(u^{0}+\varepsilon v_{1}^{0}\right)\end{array}$ & $\begin{array}{c}\text { Barrier } \\
\text { Black-Scholes }\left(u^{0}\right)\end{array}$ \\
\hline 100 & 3.587 & $3.594(0.20 \%)$ & $3.495(-2.55 \%)$ \\
102 & 2.976 & $2.987(0.39 \%)$ & $2.866(-3.68 \%)$ \\
105 & 2.170 & $2.183(0.59 \%)$ & $2.052(-5.41 \%)$ \\
\hline
\end{tabular}

$C_{\text {Barrier }}^{\mathrm{SV}, \mathcal{\varepsilon}}(T, S)$ and $C_{\text {Barrier }}^{\mathrm{BS}}(T, S)$, which confirms the validity of our method. 
TABLE 7: Case $7(c=0.0, q=0.0, \varepsilon v=0.2, \rho=-0.5, \varepsilon \lambda=0.0$, $\theta=0.0)$.

\begin{tabular}{lccc}
\hline Strike & Benchmark & $\begin{array}{c}\text { Our approximation } \\
\left(u^{0}+\varepsilon v_{1}^{0}\right)\end{array}$ & $\begin{array}{c}\text { Barrier } \\
\text { Black-Scholes }\left(u^{0}\right)\end{array}$ \\
\hline 100 & 3.261 & $3.258(-0.09 \%)$ & $3.290(0.90 \%)$ \\
102 & 2.640 & $2.639(-0.02 \%)$ & $2.686(1.78 \%)$ \\
105 & 1.841 & $1.841(0.01 \%)$ & $1.911(3.77 \%)$ \\
\hline
\end{tabular}

\section{Conclusion}

This paper has proposed an approximation scheme for barrier option prices by applying a new semigroup expansion to the Cauchy-Dirichlet problem in the second order parabolic partial differential equations (PDEs). As an application, we have derived a semigroup expansion formula under a certain type of stochastic volatility model and confirmed the validity of our method through numerical examples. Developing concrete computational schemes under various models is our next research topic.

\section{Appendices}

\section{A. Proof of Theorem 2}

First, by the definition of $v_{k}^{0}$, we easily get $v_{k}^{0}(T, x)=0$ for $x \in D$ and $v_{k}^{0}(t, x)=0$ for $(t, x) \in[0, T] \times \partial D$.

Next, fix any $x \in D$. By the Markov property, we have

$$
\begin{aligned}
& J(t\left.\wedge \tau_{D}\left(X^{0, x}\right)\right) v_{k}^{0}\left(t \wedge \tau_{D}\left(X^{0, x}\right), X_{t \wedge \tau_{D}\left(X^{0, x}\right)}^{0, x}\right) \\
&= J(t) v_{k}^{0}\left(t, X_{t}^{0, x}\right) 1_{\left\{\tau_{D}\left(X^{0, x}\right) \geq t\right\}} \\
&= \mathrm{E}\left[\int_{t}^{T \wedge \tau_{D}\left(X^{0, x}\right)} J(r) g_{k}^{0}\left(r, X_{r}^{0, x}\right) d r \mid \mathscr{F}_{t}\right] 1_{\left\{\tau_{D}\left(X^{0, x}\right) \geq t\right\}} \\
&=\mathrm{E}\left[\int_{0}^{T \wedge \tau_{D}\left(X^{0, x}\right)} J(r) g_{k}^{0}\left(r, X_{r}^{0, x}\right) d r \mid \mathscr{F}_{t}\right] \\
& \quad-\int_{0}^{t \wedge \tau_{D}\left(X^{0, x}\right)} J(r) g_{k}^{0}\left(r, X_{r}^{0, x}\right) d r
\end{aligned}
$$

for each $t \in[0, T]$, where $J(r)=\exp \left(-\int_{0}^{r} c\left(X_{v}^{0, x}, 0\right) d v\right)$ and $\left(\mathscr{F}_{r}\right)_{r}$ is the Brownian filtration. This implies that

$$
\begin{aligned}
M_{t}:=J( & \left.t \wedge \tau_{D}\left(X^{0, x}\right)\right) v_{k}^{0}\left(t \wedge \tau_{D}\left(X^{0, x}\right), X_{t \wedge \tau_{D}\left(X^{0, x}\right)}^{0, x}\right) \\
& +\int_{0}^{t \wedge \tau_{D}\left(X^{0, x}\right)} J(r) g_{k}^{0}\left(r, X_{r}^{0, x}\right) d r
\end{aligned}
$$

is a local martingale. On the other hand, applying Ito's formula, we have that

$$
\begin{aligned}
M_{t}=M_{0}+\int_{0}^{t}\left\{\left(\frac{\partial}{\partial t}+\mathscr{L}^{0}\right) v_{k}^{0}\left(r, X_{r}^{0, x}\right)\right. \\
\left.+g_{k}^{0}\left(r, X_{r}^{0, x}\right)\right\} 1_{\left\{\tau_{D}\left(X^{0, x}\right) \geq r\right\}} d r \\
+\sum_{i, j=1}^{d} \int_{0}^{t} J(r) \sigma^{i j}\left(X_{r}^{0, x}, 0\right) \frac{\partial}{\partial x^{i}} \\
\times v_{k}^{0}\left(r, X_{r}^{0, x}\right) 1_{\left\{\tau_{D}\left(X^{0, x}\right) \geq r\right\}} d B_{r}^{j}
\end{aligned}
$$

for each $t \in[0, T]$. Thus, the uniqueness of decompositions of semimartingales gives us

$$
\begin{gathered}
\int_{0}^{t}\left\{\left(\frac{\partial}{\partial t}+\mathscr{L}^{0}\right) v_{k}^{0}\left(r, X_{r}^{0, x}\right)+g_{k}^{0}\left(r, X_{r}^{0, x}\right)\right\} \\
\times 1_{\left\{\tau_{D}\left(X^{0, x}\right) \geq r\right\}} d r=0, \quad t \in[0, T] .
\end{gathered}
$$

Therefore, for each fixed $t \in(0, T)$,

$$
\begin{aligned}
\frac{1}{h} \int_{t}^{t+h} & \left\{\left(\frac{\partial}{\partial t}+\mathscr{L}^{0}\right) v_{k}^{0}\left(r, X_{r}^{0, x}\right)+g_{k}^{0}\left(r, X_{r}^{0, x}\right)\right\} \\
\times & 1_{\left\{\tau_{D}\left(X^{0, x}\right) \geq r\right\}} d r=0
\end{aligned}
$$

holds for any small enough $h>0$. Since $x \in D$, by letting $h \rightarrow 0$, we obtain

$$
\left(\frac{\partial}{\partial t}+\mathscr{L}^{0}\right) v_{k}^{0}(t, x)+g_{k}^{0}(t, x)=0
$$

Finally we prove (21) by mathematical induction. When $k=$ 0 , the assertion is easily obtained by (27), (20), (F), and (G). Now we assume that $(21)$ holds for $1,2, \ldots, k-1$. Then, by (20), (19), and (F), we have

$$
\begin{aligned}
\left|g_{k}^{0}(t, x)\right| \leq & C\left(1+|x|^{2 m}\right) \\
& \times \sum_{|\alpha| \leq 2}\left(\left|D_{\alpha} u^{0}(t, x)\right|+\sum_{l=1}^{k-1}\left|D_{\alpha} v_{l}^{0}(t, x)\right|\right)
\end{aligned}
$$

for some $C, m>0$, where $\alpha=\left(i_{1}, \ldots, i_{d}\right) \in\{0,1,2, \ldots\}^{d}$ is a multi-index, $|\alpha|=i_{1}+\cdots+i_{d}$ and $D_{\alpha}=\partial^{|\alpha|} /\left(\partial x^{1}\right)^{i_{1}} \cdots\left(\partial x^{d}\right)^{i_{d}}$. By the induction hypothesis and $(G)-(H)$, we see that

$$
\begin{gathered}
\sum_{|\alpha| \leq 2}\left(\left|D_{\alpha} u^{0}(t, x)\right|+\sum_{l=1}^{k-1}\left|D_{\alpha} v_{l}^{0}(t, x)\right|\right) \\
\leq C^{\prime} M(t)\left(1+|x|^{2 m^{\prime}}\right)
\end{gathered}
$$

for some $C^{\prime}, m^{\prime}>0$ and $M \in L^{1}([0, T) ; d t)$. Therefore, we get

$$
\left|g_{k}^{0}(t, x)\right| \leq C^{\prime \prime} M(t)\left(1+|x|^{2 m^{\prime \prime}}\right)
$$


for some $C^{\prime \prime}, m^{\prime \prime}>0$. Then we obtain

$$
\begin{aligned}
\left|v_{k}^{0}(t, x)\right| & \leq C^{\prime \prime} \mathrm{E}\left[\int_{0}^{(T-t)} M(t+r)\left(1+\left|X_{r}^{0, x}\right|^{2 m^{\prime \prime}}\right) d r\right] \\
& \leq C^{\prime \prime}\left(1+\mathrm{E}\left[\sup _{0 \leq r \leq T}\left|X_{r}^{0, x}\right|^{2 m^{\prime \prime}}\right]\right) \int_{0}^{T} M(r) d r \\
& \leq C^{\prime \prime} C_{m^{\prime \prime}} T^{m^{\prime \prime}-1}\left(\int_{0}^{T} M(r) d r\right)\left(1+|x|^{2 m^{\prime \prime}}\right)
\end{aligned}
$$

by virtue of (27). Thus (21) also holds for $k$. Now we complete the proof of Theorem 2 .

\section{B. Proof of Theorem 4}

First, we generalize the definitions of $\widetilde{\mathscr{L}}_{k}^{0}, g_{k}^{0}$, and $v_{k}^{0}$. For $k, n \geq 1$, we define

$$
\begin{aligned}
\widetilde{\mathscr{L}}_{k}^{\varepsilon}= & \frac{1}{(k-1) !} \\
& \times\left\{\frac{1}{2} \sum_{i, j=1}^{d} \int_{0}^{1}(1-r)^{k-1} \frac{\partial^{k} a^{i j}}{\partial \varepsilon^{k}}(x, r \varepsilon) d r \frac{\partial^{2}}{\partial x^{i} \partial x^{j}}\right. \\
& +\sum_{i=1}^{d} \int_{0}^{1}(1-r)^{k-1} \frac{\partial^{k} b^{i}}{\partial \varepsilon^{k}}(x, r \varepsilon) d r \frac{\partial}{\partial x^{i}} \\
& \left.-\int_{0}^{1}(1-r)^{k-1} \frac{\partial^{k} c}{\partial \varepsilon^{k}}(x, r \varepsilon) d r\right\} \\
g_{n}^{\varepsilon}(t, x)= & \widetilde{\mathscr{L}}_{n}^{\varepsilon} u^{0}(t, x)+\sum_{k=1}^{n-1} \widetilde{\mathscr{L}}_{n-k}^{0} v_{k}^{0}(t, x) \\
& +\sum_{k=1}^{n-2} \varepsilon^{k}\left\{\widetilde{\mathscr{L}}_{n}^{\varepsilon} v_{k}^{0}(t, x)+\sum_{l=k+1}^{n-1} \widetilde{\mathscr{L}}_{n+k-l}^{0} v_{l}^{0}(t, x)\right\} \\
& +\varepsilon^{n-1} \widetilde{\mathscr{L}}_{n}^{\varepsilon} v_{n-1}^{0}(t, x),
\end{aligned}
$$

where $g_{1}^{\varepsilon}(t, x)$ and $g_{2}^{\varepsilon}(t, x)$ are understood as $g_{1}^{\varepsilon}(t, x)=$ $\widetilde{\mathscr{L}}_{1}^{\varepsilon} u^{0}(t, x)$ and $g_{2}^{\varepsilon}(t, x)=\widetilde{\mathscr{L}}_{2}^{\varepsilon} u^{0}(t, x)+\widetilde{\mathscr{L}}_{1}^{0} v_{1}^{0}(t, x)+$ $\varepsilon \widetilde{\mathscr{L}}_{2}^{\varepsilon} v_{1}^{0}(t, x)$, respectively.

We consider the following Cauchy-Dirichlet problem:

$$
\begin{gathered}
-\frac{\partial}{\partial t} v(t, x)-\mathscr{L}^{\varepsilon} v(t, x)-g_{n}^{\varepsilon}(t, x)=0, \\
(t, x) \in[0, T) \times D, \\
v(T, x)=0, \quad x \in D, \\
v(t, x)=0, \quad(t, x) \in[0, T] \times \partial D .
\end{gathered}
$$

For $\varepsilon \neq 0$, we define $v_{n}^{\varepsilon}=\left[u^{\varepsilon}-\left\{u^{0}+\sum_{k=1}^{n-1} \varepsilon^{k} v_{k}^{0}(t, x)\right\}\right] / \varepsilon^{n}$. Obviously, we see

$$
u^{\varepsilon}(t, x)=u^{0}(t, x)+\sum_{k=1}^{n-1} \varepsilon^{k} v_{k}^{0}(t, x)+\varepsilon^{n} v_{n}^{\varepsilon}(t, x) .
$$

Proposition 16. The function $v_{n}^{\varepsilon}$ is a solution of (B.2).

Proof. It is obvious that $v_{n}^{\varepsilon}(T, x)=0$ for $x \in D$ and $v_{n}^{\varepsilon}(t, x)=$ 0 for $(t, x) \in[0, T] \times \partial D$.

We also recall in (14) that

$$
\frac{\partial}{\partial t} u^{\varepsilon}(t, x)+\mathscr{L}^{\varepsilon} u^{\varepsilon}(t, x)=0 .
$$

Next, we apply Taylor's theorem to $\mathscr{L}^{\varepsilon}$ to observe that

$$
\mathscr{L}^{\varepsilon} u^{\varepsilon}(t, x)=\left\{\mathscr{L}^{0}+\sum_{k=1}^{n-1} \varepsilon^{k} \widetilde{\mathscr{L}}_{k}^{0}+\varepsilon^{n} \widetilde{\mathscr{L}}_{n}^{\varepsilon}\right\} u^{\varepsilon}(t, x)
$$

Since $u^{0}$ is the solution of (14) with $\varepsilon=0$, we get

$$
\frac{\partial}{\partial t} u^{0}(t, x)+\mathscr{L}^{0} u^{0}(t, x)=0
$$

Similarly, since $v_{k}^{0}$ is a solution of (18), we have

$$
\frac{\partial}{\partial t} v_{k}^{0}(t, x)+\mathscr{L}^{0} v_{k}^{0}(t, x)+\widetilde{\mathscr{L}}_{k}^{0} u^{0}(t, x)+\sum_{l=1}^{k-1} \widetilde{\mathscr{L}}_{k-l}^{0} v_{l}^{0}(t, x)=0
$$

Then, replacing $u^{\varepsilon}(t, x)$ in the right hand side of (B.5) by the right hand side of (B.3) and taking (B.6) and (B.7) into account, we obtain

$$
\begin{aligned}
& \varepsilon^{n}\left\{\frac{\partial}{\partial t} v_{n}^{\varepsilon}(t, x)+\mathscr{L}^{0} v_{n}^{\varepsilon}(t, x)\right. \\
& \left.+\widetilde{\mathscr{L}}_{n}^{\varepsilon} u^{0}(t, x)+\sum_{l=1}^{n-1} \widetilde{\mathscr{L}}_{n-l}^{0} v_{l}^{0}(t, x)\right\} \\
& +\sum_{k=n+1}^{2 n-2} \varepsilon^{k}\left\{\widetilde{\mathscr{L}}_{k-n}^{0} v_{n}^{\varepsilon}(t, x)+\widetilde{\mathscr{L}}_{n}^{\varepsilon} v_{k-n}^{0}(t, x)\right. \\
& \left.+\sum_{l=k-n+1}^{n-1} \widetilde{\mathscr{L}}_{k-l}^{0} v_{l}^{0}(t, x)\right\} \\
& +\varepsilon^{2 n-1}\left\{\widetilde{\mathscr{L}}_{n-1}^{0} v_{n}^{\varepsilon}(t, x)+\widetilde{\mathscr{L}}_{n}^{\varepsilon} v_{n-1}^{\varepsilon}(t, x)\right\}
\end{aligned}
$$

Thus, with the definition of $g_{n}^{\varepsilon}(t, x)$ above, we have for $\varepsilon \neq 0$,

$$
\frac{\partial}{\partial t} v_{n}^{\varepsilon}(t, x)+\mathscr{L}^{\varepsilon} v_{n}^{\varepsilon}(t, x)+g_{n}^{\varepsilon}(t, x)=0 .
$$

This implies the assertion. 
Set

$$
\begin{aligned}
& \tilde{v}_{n}^{\varepsilon}(t, x)=\mathrm{E}\left[\int_{0}^{\tau_{D}\left(X^{\varepsilon, x}\right) \wedge(T-t)}\right. \exp \left(-\int_{0}^{r} c\left(X_{v}^{\varepsilon, x}, \varepsilon\right) d v\right) \\
&\left.\times g_{n}^{\varepsilon}\left(t+r, X_{r}^{\varepsilon, x}\right) d r\right] .
\end{aligned}
$$

By (G)-(H), we find that there are $C_{n}>0, \widetilde{m}_{n} \in \mathbb{N}$ which are independent of $\varepsilon$ and the function $M_{n} \in C([0, T)) \cap$ $L^{1}([0, T), d t)$ determined by $u^{0}, v_{1}^{0}, \ldots, v_{n-1}^{0}$ such that

$$
\left|g_{n}^{\varepsilon}(t, x)\right| \leq C_{n} M_{n}(t)\left(1+|x|^{2 \widetilde{m}_{n}}\right) .
$$

The inequalities (27) and (B.11) imply

$$
\left|\widetilde{v}_{n}^{\varepsilon}(t, x)\right| \leq C_{n}^{\prime} \int_{t}^{T} M_{n}(r) d r\left(1+|x|^{2 \widetilde{m}_{n}}\right)
$$

for some $C_{n}^{\prime}>0$ which is also independent of $\varepsilon$.

Proposition 17. $v_{n}^{\varepsilon}=\widetilde{v}_{n}^{\varepsilon}$.

Proof. The assertion is easily obtained by the similar argument to the one in Theorem 5.1.9 in [20].

Proof of Theorem 4. By (B.3) and Proposition 17, we have $u^{\varepsilon}(t, x)-\left(u^{0}(t, x)+\sum_{k=1}^{n-1} \varepsilon^{k} v_{k}^{0}(t, x)\right)=\varepsilon^{n} \widetilde{v}_{n}^{\varepsilon}(t, x)$. Our assertion is now immediately obtained by inequality (B.12).

\section{Proof of Theorem 7}

(1) Firstly, let us consider the case for $k=1$. Let $g \in \mathscr{H}^{m, 1}$. Observe that

$$
\begin{aligned}
& \int_{0}^{(T-t) \wedge \tau_{D}\left(X^{0, x}\right)} \exp \left(-\int_{0}^{r} c\left(X_{v}^{0, x}, 0\right) d v\right) g\left(t+r, X_{r}^{0, x}\right) d r \\
&=\int_{0}^{T-t} \exp \left(-\int_{0}^{r} c\left(X_{v}^{0, x}, 0\right) d v\right) \\
& \times g\left(t+r, X_{r}^{0, x}\right) 1_{\left\{\tau_{D}\left(X^{0, x}\right) \geq r\right\}} d r
\end{aligned}
$$

and we obtain

$$
\begin{gathered}
\mathrm{E}\left[\int_{0}^{(T-t) \wedge \tau_{D}\left(X^{0, x}\right)} \exp \left(-\int_{0}^{r} c\left(X_{v}^{0, x}, 0\right) d v\right)\right. \\
\left.\times g\left(t+r, X_{r}^{0, x}\right) d r\right] \\
=\int_{0}^{T-t} \mathrm{E}\left[\exp \left(-\int_{0}^{r} c\left(X_{v}^{0, x}, 0\right) d v\right)\right. \\
\left.\times g\left(t+r, X_{r}^{0, x}\right) 1_{\left\{\tau_{D}\left(X^{0, x}\right) \geq r\right\}}\right] d r \\
=\int_{0}^{T-t} P_{r}^{D} g(t+r, \cdot)(x) d r .
\end{gathered}
$$

Thus, under the assumption $(\mathrm{H})$, we see

$$
\begin{aligned}
& v_{1}^{0}(T-t, x) \\
& =\mathrm{E}\left[\int_{0}^{t} \exp \left(-\int_{0}^{r} c\left(X_{v}^{0, x}, 0\right) d v\right)\right. \\
& \left.\quad \times g_{1}^{0}\left(T-t+r, X_{r}^{0, x}\right) 1_{\left\{\tau_{D}\left(X^{0, x}\right) \geq r\right\}} d r\right] \\
& =\int_{0}^{t} P_{r}^{D} \widetilde{\mathscr{L}}_{1}^{0} u^{0}(T-t+r, \cdot)(x) d r \\
& =\int_{0}^{t} P_{r}^{D} \widetilde{\mathscr{L}}_{1}^{0} P_{t-r}^{D} f(x) d r=\int_{0}^{t} P_{t-r}^{D} \widetilde{\mathscr{L}}_{1}^{0} P_{r}^{D} f(x) d r .
\end{aligned}
$$

Thus, we have the assertion for $k=1$.

(2) If the assertion holds for $1, \ldots, k-1$, then

$$
\begin{aligned}
& v_{k}^{0}(T-t, x) \\
& =\int_{0}^{t} P_{t_{0}}^{D}\left\{\widetilde{\mathscr{L}}_{k}^{0} u^{0}+\sum_{l=1}^{k-1} \widetilde{\mathscr{L}}_{k-l}^{0} v_{l}^{0}\right\}\left(T-t+t_{0}, \cdot\right)(x) d t_{0} \\
& =\int_{0}^{t} P_{t-t_{0}}^{D} \widetilde{\mathscr{L}}_{k}^{0} P_{t_{0}}^{D} f(x) d t_{0} \\
& \quad+\sum_{l=1}^{k-1} \sum_{m=1}^{l} \sum_{\left(\beta^{i}\right)_{i=1}^{m} \mathbb{N}^{m}, \sum_{i} \beta^{i}=l} \int_{0}^{t} \int_{0}^{t_{0}} \int_{0}^{t_{1}} \cdots \int_{0}^{t_{l-1}} P_{t-t_{0}}^{D} \widetilde{\mathscr{L}}_{k-l}^{0} P_{t_{0}-t_{1}}^{D} \widetilde{\mathscr{L}}_{\beta^{1}}^{0} P_{t_{1}-t_{2}}^{D} \widetilde{\mathscr{L}}_{\beta^{2}}^{0} \cdots P_{t_{l-1}-t_{l}}^{D} \widetilde{\mathscr{L}}_{\beta^{l}}^{0} P_{t_{l}}^{D} f(x) d t_{l} \cdots d t_{1} d t_{0} \\
& =\int_{0}^{t} P_{t-t_{0}}^{D} \widetilde{\mathscr{L}}_{k}^{0} P_{t_{0}}^{D} f(x) d t_{0}
\end{aligned}
$$




$$
\begin{aligned}
& +\sum_{l=2}^{k} \sum_{m=1}^{l} \sum_{\left(\beta^{i}\right)_{i=1}^{m} \subset \mathbb{N}^{m}, \sum_{i} \beta^{i}=k} \int_{0}^{t} \int_{0}^{t_{1}} \int_{0}^{t_{2}} \cdots \int_{0}^{t_{l-1}} P_{t-t_{1}}^{D} \widetilde{\mathscr{L}}_{\beta^{1}}^{0} P_{t_{1}-t_{2}}^{D} \widetilde{\mathscr{L}}_{\beta^{2}}^{0} P_{t_{2}-t_{3}}^{D} \widetilde{\mathscr{L}}_{\beta^{3}}^{0} \cdots P_{t_{l-1}-t_{l}}^{D} \widetilde{\mathscr{L}}_{\beta^{l}}^{0} P_{t_{l}}^{D} f(x) d t_{l} \cdots d t_{1} \\
= & \sum_{l=1}^{k} \sum_{\left(\beta^{i}\right)_{i=1}^{l} \subset \mathbb{N}^{l}, \sum_{i} \beta^{i}=k} \int_{0}^{t} \int_{0}^{t_{1}} \cdots \int_{0}^{t_{l-1}} P_{t-t_{1}}^{D} \widetilde{\mathscr{L}}_{\beta^{1}}^{0} P_{t_{1}-t_{2}}^{D} \widetilde{\mathscr{L}}_{\beta^{2}}^{0} \cdots P_{t_{l-1}-t_{l}}^{D} \widetilde{\mathscr{L}}_{\beta^{l}}^{0} P_{t_{l}}^{D} f(x) d t_{l} \cdots d t_{1} .
\end{aligned}
$$

Thus, our assertion is also true for $k$. Then we complete the proof of Proposition 17 by mathematical induction.

\section{Proof of Theorem 15}

By the asymptotic expansion in Section 2 and Theorem 7 with $k=1$, we see that the expansion

$$
C_{\text {Barrier }}^{\mathrm{SV}, \varepsilon}\left(T, e^{x}\right)=C_{\text {Barrier }}^{\mathrm{BS}, \varepsilon}\left(T, e^{x}\right)+\varepsilon v_{1}^{0}(0, x)+O\left(\varepsilon^{2}\right)
$$

holds with

$$
v_{1}^{0}(t, x)=\int_{0}^{T-t} P_{T-t-r}^{D} \widetilde{\mathscr{L}}_{1}^{0} P_{r}^{D \bar{f}}(x) d r
$$

Then, we have the following proposition for an expression of $v_{1}^{0}(0, x)$. The proof is given in Appendix D.1.

Proposition 18. It holds that:

$$
\begin{aligned}
v_{1}^{0}(0, x)= & \frac{T}{2} \nu \rho \sigma^{2} \frac{\partial^{2}}{\partial x \partial \sigma} P_{T}^{D \bar{f}}(x) \\
-\frac{1}{2} E[ & {\left[\left(T-\tau_{D}\left(X^{0, x}\right)\right) \nu \rho \sigma^{2} \frac{\partial^{2}}{\partial x \partial \sigma}\right.} \\
& \left.\times P_{T-\tau_{D}\left(X^{0, x}\right)}^{D} \bar{f}(l) 1_{\left\{\tau_{D}\left(X^{0, x}\right)<T\right\}}\right] .
\end{aligned}
$$

We remark that the expectation in the above equality can be represented as

$$
\begin{gathered}
\frac{1}{2} E\left[\left(T-\tau_{D}\left(X^{0, x}\right)\right) \nu \rho \sigma^{2} \frac{\partial^{2}}{\partial x \partial \sigma} P_{T-\tau_{D}\left(X^{0, x}\right)}^{D} \bar{f}(l) 1_{\left\{\tau_{D}\left(X^{0, x}\right)<T\right\}}\right] \\
\quad=\int_{0}^{T} \frac{(T-s)}{2} \nu \rho \sigma^{2} \frac{\partial^{2}}{\partial x \partial \sigma} P_{T-s}^{D} \bar{f}(l) h(s, x-l) d s,
\end{gathered}
$$

where $h(s, x-l)$ is the density function of the first hitting time to $l$ defined by

$$
h(s, x-l)=\frac{-(l-x)}{\sqrt{2 \pi \sigma^{2} s^{3}}} \exp \left(-\frac{\left\{l-x+\sigma^{2} s / 2\right\}^{2}}{2 \sigma^{2} s}\right) .
$$

Now we evaluate

$$
\begin{aligned}
\nu \rho \sigma^{2} \frac{\partial^{2}}{\partial x \partial \sigma} P_{t}^{D} \bar{f}(x)= & \nu \rho \sigma^{2} \frac{\partial^{2}}{\partial x \partial \sigma} C^{B S}\left(t, e^{x}\right) \\
& -v \rho \sigma^{2} \frac{\partial^{2}}{\partial x \partial \sigma}\left\{\left(\frac{e^{x}}{L}\right) C^{B S}\left(t, \frac{L^{2}}{e^{x}}\right)\right\} .
\end{aligned}
$$

Note that

$$
\begin{gathered}
\frac{\partial}{\partial \sigma} C^{B S}\left(t, e^{x}\right)=e^{x} n\left(d_{1}(t, x)\right) \sqrt{t} \\
\frac{\partial}{\partial \sigma}\left\{\left(\frac{e^{x}}{L}\right) C^{B S}\left(t, \frac{L^{2}}{e^{x}}\right)\right\}=\operatorname{Ln}\left(c_{1}(t, x)\right) \sqrt{t}
\end{gathered}
$$

Then we have

$$
\begin{aligned}
& v \rho \sigma^{2} \frac{\partial^{2}}{\partial x \partial \sigma} C^{B S}\left(t, e^{x}\right) \\
& =v \rho \sigma^{2} e^{x} n\left(d_{1}(t, x)\right) \sqrt{t}\left\{1-\frac{d_{1}(t, x)}{\sigma \sqrt{t}}\right\} \\
& =-v \rho \sigma e^{x} n\left(d_{1}(t, x)\right) d_{2}(t, x), \\
& v \rho \sigma^{2} \frac{\partial^{2}}{\partial x \partial \sigma}\left\{\left(\frac{e^{x}}{L}\right) C^{B S}\left(t, \frac{L^{2}}{e^{x}}\right)\right\} \\
& \quad=v \rho \sigma \operatorname{Ln}\left(c_{1}(t, x)\right) c_{1}(t, x) .
\end{aligned}
$$

Combining (D.7) and (D.9), we get

$$
\begin{aligned}
& v \rho \sigma^{2} \frac{\partial^{2}}{\partial x \partial \sigma} P_{t}^{D} \bar{f}(x) \\
& \quad=v \rho \sigma\left\{e^{x} n\left(d_{1}(t, x)\right)\left(-d_{2}(t, x)\right)-\operatorname{Ln}\left(c_{1}(t, x)\right) c_{1}(t, x)\right\} .
\end{aligned}
$$


Substituting (D.10) into (D.4), we have

$$
\begin{aligned}
& \nu \rho \sigma^{2} \frac{\partial^{2}}{\partial x \partial \sigma} P_{t}^{D} \bar{f}(l) \\
& =v \rho \sigma \operatorname{Ln}\left(d_{1}(t, l)\right)\left(-d_{2}(t, l)\right)-\rho \sigma \operatorname{Ln}\left(c_{1}(t, l)\right) c_{1}(t, l) \\
& =v \rho \sigma \operatorname{Ln}\left(d_{1}(t, l)\right)\left(-\left(d_{1}(t, l)+d_{2}(t, l)\right)\right) \\
& =v \rho \sigma \frac{1}{\sqrt{2 \pi}} \exp \left(-\frac{\left\{l-\log K+(1 / 2) \sigma^{2} t\right\}^{2}}{2 \sigma^{2} t}\right) \\
& \quad \times\left(\frac{-2(l-\log K)}{\sigma \sqrt{t}}\right) .
\end{aligned}
$$

Thus we obtain

$$
\begin{aligned}
& -\frac{1}{2} E\left[\left(T-\tau_{D}\left(X^{0, x}\right)\right) \nu \rho \sigma^{2} \frac{\partial^{2}}{\partial x \partial \sigma}\right. \\
& \left.\quad \times P_{T-\tau_{D}\left(X^{0, x}\right)}^{\bar{f}}(l) 1_{\left\{\tau_{D}\left(X^{0, x}\right)<T\right\}}\right] \\
& =-\int_{0}^{T} \frac{(T-s)}{2} v \rho \sigma L \frac{1}{\sqrt{2 \pi}} e^{-\left\{l-\log K+(1 / 2) \sigma^{2}(T-s)\right\}^{2} / 2 \sigma^{2}(T-s)} \\
& \quad \times\left(\frac{-2(l-\log K)}{\sigma \sqrt{T-s}}\right) \\
& \quad \times \frac{-(l-x)}{\sqrt{2 \pi \sigma^{2} s^{3}}} e^{-\left\{(l-x)+\left(\sigma^{2} / 2\right) s\right\}^{2} / 2 \sigma^{2} s} d s \\
& =\frac{\nu \rho L(x-l) \log (L / K)}{2 \pi \sigma} \\
& \quad \times \int_{0}^{T} \frac{(T-s)^{1 / 2}}{s^{3 / 2}} \exp \left(-\frac{c_{2}(T-s, L / K)+c_{2}\left(s, L / e^{x}\right)}{2}\right) d s .
\end{aligned}
$$

By Proposition 18, (D.4), (D.10), and (D.12), we reach the assertion.

D.1. Proof of Proposition 18. First, we notice the following relation:

$$
\widetilde{\mathscr{L}}_{1}^{0} P_{t}^{D} \bar{f}(x)=v \rho \sigma^{3} t\left(\frac{\partial^{3}}{\partial x^{3}}-\frac{\partial^{2}}{\partial x^{2}}\right) P_{t}^{D \bar{f}}(x) .
$$

Then, using the relations $\mathscr{L}^{0} \widetilde{\mathscr{L}}_{1}^{0} P_{t}^{D} \bar{f}(x)=\widetilde{\mathscr{L}}_{1}^{0} \mathscr{L}^{0} P_{t}^{D} \bar{f}(x)$ and

$$
\left(\frac{\partial}{\partial t}+\mathscr{L}^{0}\right) P_{T-t}^{D} \bar{f}(x)=0
$$

we get

$$
\left(\frac{\partial}{\partial t}+\mathscr{L}^{0}\right) \frac{T-t}{2} \widetilde{\mathscr{L}}_{1}^{0} P_{T-t}^{D} \bar{f}(x)=-\widetilde{\mathscr{L}}_{1}^{0} P_{T-t}^{D} \bar{f}(x) .
$$

Also, we have

$$
\begin{aligned}
& \left(\frac{\partial}{\partial t}+\mathscr{L}^{0}\right) \int_{0}^{T-t} P_{T-t-r}^{D}\left(\nu \rho \sigma^{2} \frac{\partial^{2}}{\partial x \partial \sigma} P_{r}^{D} \bar{f}\right)(x) d r \\
& =-\widetilde{\mathscr{L}}_{1}^{0} P_{T-t}^{D} \bar{f}(x), \quad x \in(l, \infty) .
\end{aligned}
$$

Therefore, the function

$$
\begin{aligned}
\eta(t, x)= & \int_{0}^{T-t} P_{T-t-r}^{D}\left(\nu \rho \sigma^{2} \frac{\partial^{2}}{\partial x \partial \sigma} P_{r}^{D} \bar{f}\right)(x) d r \\
& -\frac{T-t}{2} \widetilde{\mathscr{L}}_{1}^{0} P_{T-t}^{D} \bar{f}(x)
\end{aligned}
$$

satisfies the following PDE

$$
\begin{gathered}
\left(\frac{\partial}{\partial t}+\mathscr{L}^{0}\right) \eta(t, x)=0, \quad(t, x) \in[0, T) \times(l, \infty), \\
\eta(T, x)=0, \quad x \in[l, \infty) \\
\eta(t, l)=-\frac{T-t}{2} \widetilde{\mathscr{L}}_{1}^{0} P_{T-t}^{D} \bar{f}(l), \quad t \in[0, T) .
\end{gathered}
$$

Then Theorem 6.5.2 in [9] implies

$$
\begin{aligned}
\eta(0, x)=-\frac{1}{2} \mathrm{E}[ & \left(T-\tau_{D}\left(X^{0, x}\right)\right) \nu \rho \sigma^{2} \frac{\partial^{2}}{\partial x \partial \sigma} \\
& \left.\times P_{T-\tau_{D}\left(X^{0, x}\right)}^{D} \bar{f}(l) 1_{\left\{\tau_{D}\left(X^{0, x}\right)<T\right\}}\right] .
\end{aligned}
$$

By (D.17) and (D.19), we get the assertion.

\section{Disclosure}

Mathematical Subject Classification (2010) 35B20, 35C20, 91G20.

\section{Conflict of Interests}

The authors declare that there is no conflict of interests regarding the publication of this paper.

\section{Acknowledgments}

The authors are very grateful to Professor Seisho Sato in the University of Tokyo for his suggestions to our numerical computation. Also we would like to thank the anonymous referees for their valuable comments and suggestions.

\section{References}

[1] R. C. Merton, "Theory of rational option pricing," Bell Journal of Economics and Management Science, vol. 4, no. 1, pp. 141-183, 1973.

[2] E. Gobet, "Weak approximation of killed diffusion using Euler schemes," Stochastic Processes and their Applications, vol. 87, no. 2, pp. 167-197, 2000. 
[3] H. Pham, Large deviations in Finance, Third SMAI European Summer School in Financial Mathematics, 2010.

[4] E. Fournié, J.-M. Lasry, J. Lebuchoux, and P.-L. Lions, "Applications of Malliavin calculus to Monte-Carlo methods in finance. II," Finance and Stochastics, vol. 5, no. 2, pp. 201-236, 2001.

[5] N. Kunitomo and A. Takahashi, "On validity of the asymptotic expansion approach in contingent claim analysis," The Annals of Applied Probability, vol. 13, no. 3, pp. 914-952, 2003.

[6] A. Takahashi and T. Yamada, "An asymptotic expansion with push-down of Malliavin weights," SIAM Journal on Financial Mathematics, vol. 3, no. 1, pp. 95-136, 2012.

[7] K. Shiraya, A. Takahashi, and T. Yamada, "Pricing discrete barrier options under stochastic volatility," Asia-Pacific Financial Markets, vol. 19, no. 3, pp. 205-232, 2012.

[8] A. Friedman, Partial Differential Equations of Parabolic Type, Prentice-Hall, 1964.

[9] A. Friedman, Stochastic Differential Equations and Applications, vol. 1, Academic Press, 1975.

[10] G. M. Lieberman, Second Order Parabolic Differential Equations, World Scientific, River Edge, NJ, USA, 1996.

[11] G. Rubio, "The Cauchy-Dirichlet problem for a class of linear parabolic differential equations with unbounded coefficients in an unbounded domain," International Journal of Stochastic Analysis, vol. 2011, Article ID 469806, 35 pages, 2011.

[12] J. P. Fouque, G. Papanicolaou, and K. R. Sircar, "Mean reverting stochastic volatility," International Journal of Theoretical and Applied Finance, vol. 3, pp. 101-142, 2000.

[13] J. Fouque, G. Papanicolaou, and K. R. Sircar, Derivatives in Financial Markets with Stochastic Volatility, Cambridge University Press, Cambridge, UK, 2000.

[14] S. Howison and M. Steinberg, "A matched asymptotic expansions approach to continuity corrections for discretely sampled options, I: barrier options," Applied Mathematical Finance, vol. 14, no. 1, pp. 63-89, 2007.

[15] A. Ilhan, M. Jonsson, and R. Sircar, "Singular perturbations for boundary value problems arising from exotic options," SIAM Journal on Applied Mathematics, vol. 64, no. 4, pp. 1268-1293, 2004.

[16] I. Karatzas and S. E. Shreve, Brownian Motion and Stochastic Calculus, Springer, New York, NY, USA, 2nd edition, 1991.

[17] N. V. Krylov, Controlled Diffusion Processes, Springer, Berlin, Germany, 1980.

[18] H. Nagai, Stochastic Differential Equations, Kyoritsu Shuppan, 1999 (Japanese).

[19] M. G. Crandall, H. Ishii, and P. Lions, "User's guide to viscosity solutions of second order partial differential equations," The American Mathematical Society. Bulletin. New Series, vol. 27, no. 1, pp. 1-67, 1992.

[20] D. Lamberton and B. Lapeyre, Introduction to Stochastic Calculus Applied to Finance, translated by N. Rabeau and F. Mantion, Chapman \& Hall/CRC, New York, USA, 1996. 


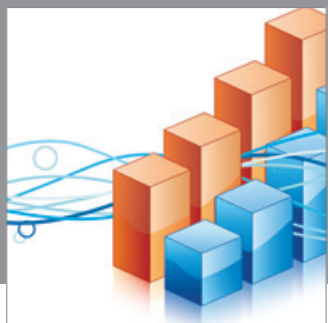

Advances in

Operations Research

mansans

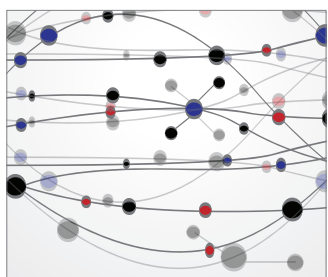

The Scientific World Journal
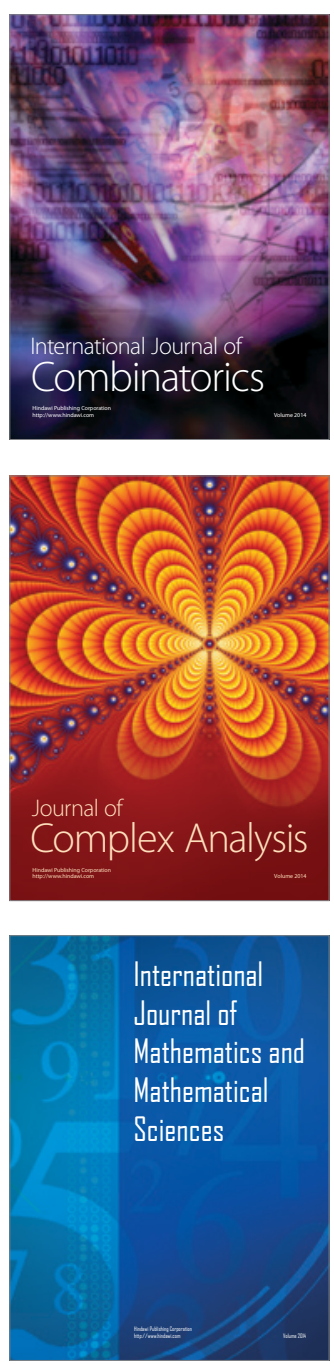
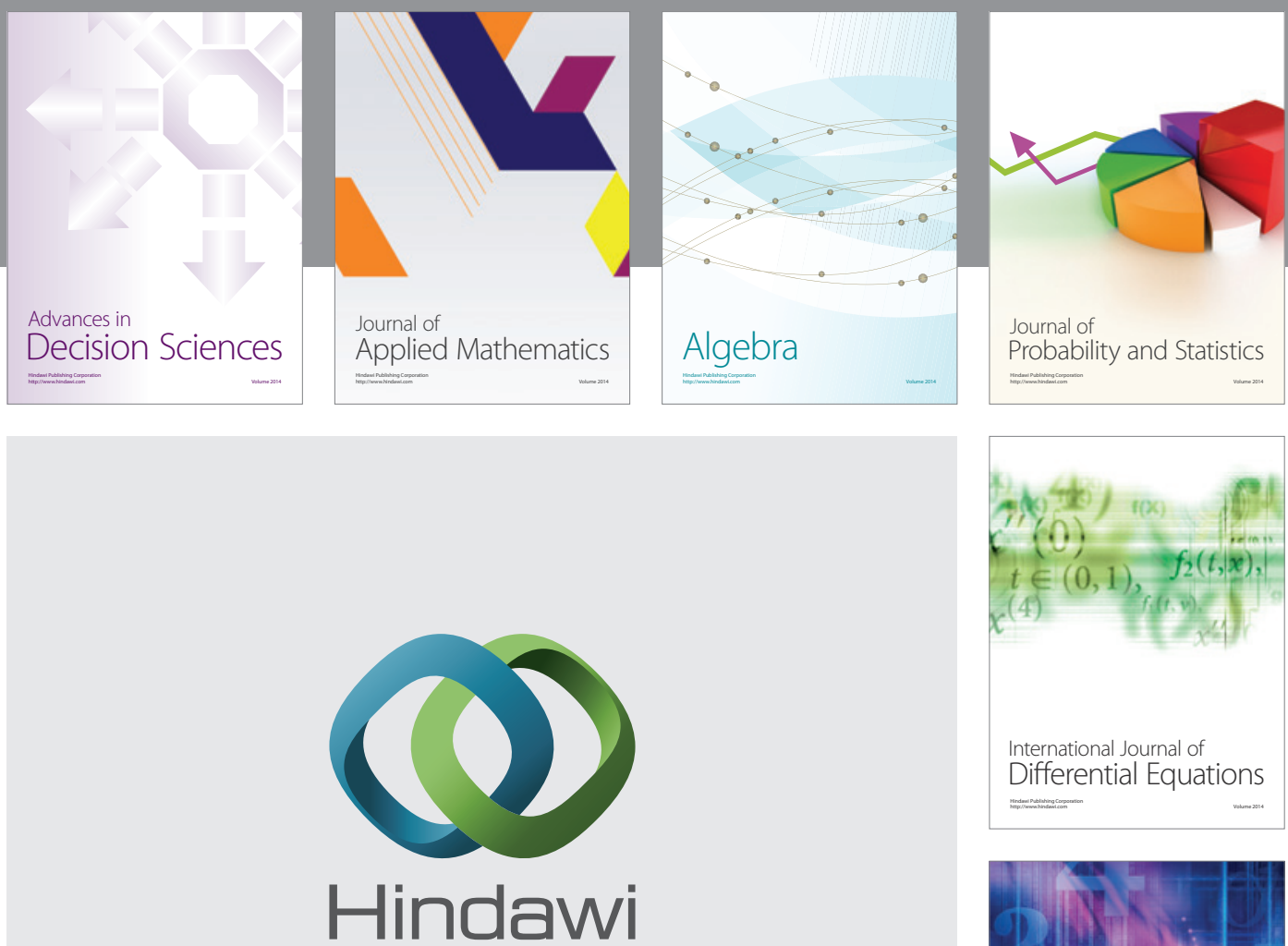

Submit your manuscripts at http://www.hindawi.com
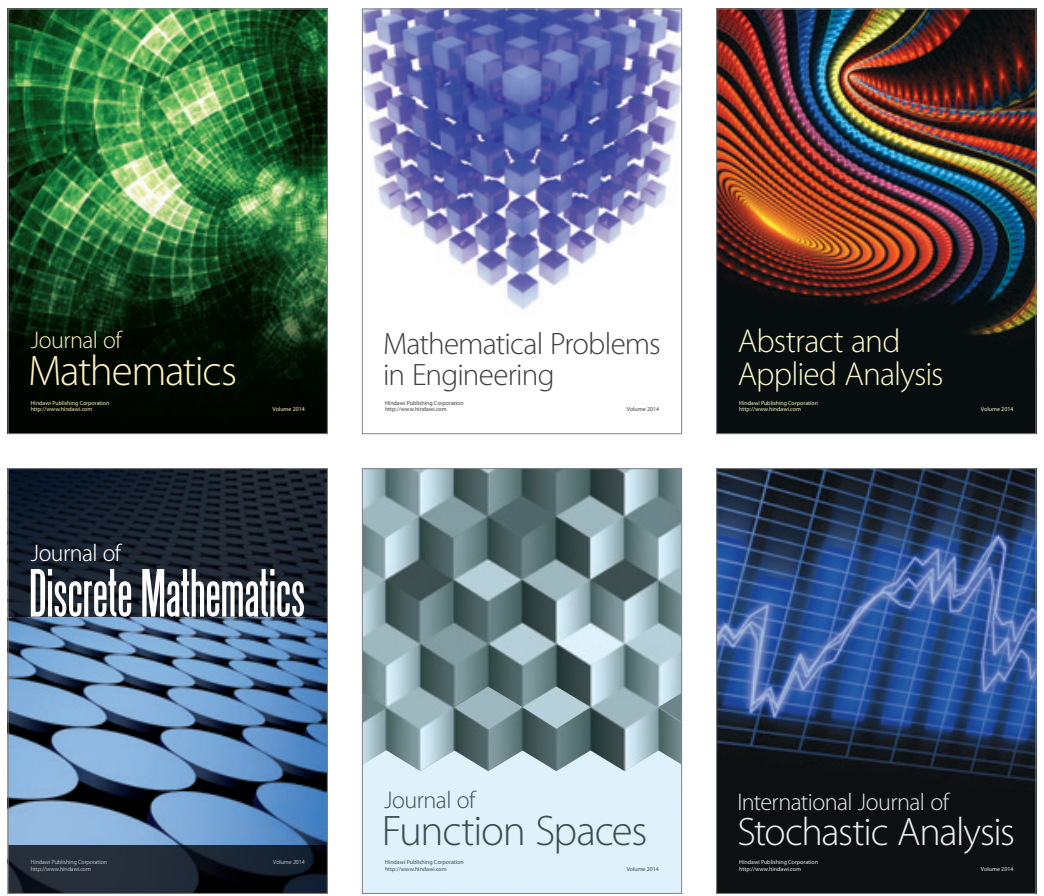

Journal of

Function Spaces

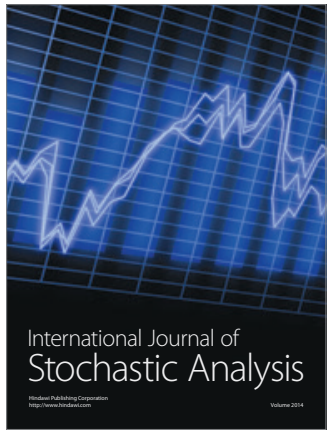

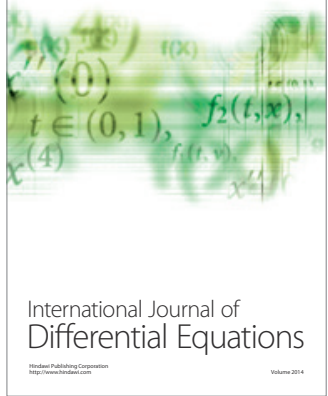
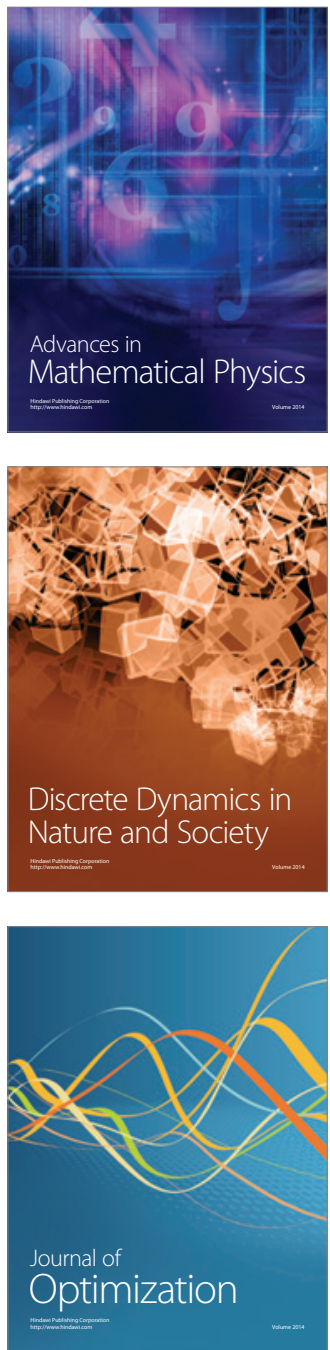